Atmos. Chem. Phys., 18, 15169-15182, 2018

https://doi.org/10.5194/acp-18-15169-2018

(C) Author(s) 2018. This work is distributed under

the Creative Commons Attribution 4.0 License.

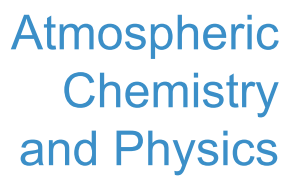

(c) (P)

\title{
Emissions from village cookstoves in Haryana, India, and their potential impacts on air quality
}

\author{
Lauren T. Fleming ${ }^{1}$, Robert Weltman ${ }^{2}$, Ankit Yadav ${ }^{3}$, Rufus D. Edwards ${ }^{2}$, Narendra K. Arora ${ }^{3}$, Ajay Pillarisetti ${ }^{4}$, \\ Simone Meinardi ${ }^{1}$, Kirk R. Smith ${ }^{4}$, Donald R. Blake ${ }^{1}$, and Sergey A. Nizkorodov ${ }^{1}$ \\ ${ }^{1}$ Department of Chemistry, University of California, Irvine, CA 92697, USA \\ ${ }^{2}$ Department of Epidemiology, University of California, Irvine, CA 92697, USA \\ ${ }^{3}$ The Inclen Trust, Okhla Industrial Area, Phase-I, New Delhi-110020, India \\ ${ }^{4}$ School of Public Health, University of California, Berkeley, CA 94720, USA
}

Correspondence: Sergey A. Nizkorodov (nizkorod@uci.edu)

Received: 15 May 2018 - Discussion started: 8 June 2018

Revised: 4 October 2018 - Accepted: 5 October 2018 - Published: 22 October 2018

\begin{abstract}
Air quality in rural India is impacted by residential cooking and heating with biomass fuels. In this study, emissions of $\mathrm{CO}, \mathrm{CO}_{2}$, and 76 volatile organic compounds (VOCs) and fine particulate matter $\left(\mathrm{PM}_{2.5}\right)$ were quantified to better understand the relationship between cook fire emissions and ambient ozone and secondary organic aerosol (SOA) formation. Cooking was carried out by a local cook, and traditional dishes were prepared on locally built chulha or angithi cookstoves using brushwood or dung fuels. Cook fire emissions were collected throughout the cooking event in a Kynar bag (VOCs) and on polytetrafluoroethylene (PTFE) filters $\left(\mathrm{PM}_{2.5}\right)$. Gas samples were transferred from a Kynar bag to previously evacuated stainless-steel canisters and analyzed using gas chromatography coupled to flame ionization, electron capture, and mass spectrometry detectors. VOC emission factors were calculated from the measured mixing ratios using the carbon-balance method, which assumes that all carbon in the fuel is converted to $\mathrm{CO}_{2}, \mathrm{CO}$, VOCs, and $\mathrm{PM}_{2.5}$ when the fuel is burned. Filter samples were weighed to calculate $\mathrm{PM}_{2.5}$ emission factors. Dung fuels and angithi cookstoves resulted in significantly higher emissions of most VOCs $(p<0.05)$. Utilizing dung-angithi cook fires resulted in twice as much of the measured VOCs compared to dungchulha and 4 times as much as brushwood-chulha, with 84.0, 43.2 , and $17.2 \mathrm{~g}$ measured $\mathrm{VOC} \mathrm{kg}{ }^{-1}$ fuel carbon, respectively. This matches expectations, as the use of dung fuels and angithi cookstoves results in lower modified combustion efficiencies compared to brushwood fuels and chulha cookstoves. Alkynes and benzene were exceptions and had
\end{abstract}

significantly higher emissions when cooking using a chulha as opposed to an angithi with dung fuel (for example, benzene emission factors were $3.18 \mathrm{~g} \mathrm{~kg}^{-1}$ fuel carbon for dungchulha and $2.38 \mathrm{~g} \mathrm{~kg}^{-1}$ fuel carbon for dung-angithi). This study estimated that 3 times as much SOA and ozone in the maximum incremental reactivity (MIR) regime may be produced from dung-chulha as opposed to brushwood-chulha cook fires. Aromatic compounds dominated as SOA precursors from all types of cook fires, but benzene was responsible for the majority of SOA formation potential from all chulha cook fire VOCs, while substituted aromatics were more important for dung-angithi. Future studies should investigate benzene exposures from different stove and fuel combinations and model SOA formation from cook fire VOCs to verify public health and air quality impacts from cook fires.

\section{Introduction}

Parts of rural India are comprised of densely populated villages with ambient ozone and $\mathrm{PM}_{2.5}$ levels that affect air quality for inhabitants (Bisht et al., 2015; Ojha et al., 2012; Reddy, 2012). For example, in the rural area of Anantapur in southern India, monthly mean ozone levels varied between 29 ppbv (parts per billion by volume) in August during the monsoon season and 56 ppbv in April (Reddy, 2012). In Pantnagar, a semi-urban city, the maximum observed ozone concentration was $105 \mathrm{ppbv}$ for one day in May, with the lowest maximum of 50 ppbv being in January (Ojha et al., 2012). In 
terms of $\mathrm{PM}_{2.5}$ levels, Bisht et al. (2015) observed an average of $50 \mu \mathrm{g} \mathrm{m}^{-3}$ of $\mathrm{PM}_{2.5}$ over July-November 2011 in rural Mahabubnagar. While measurements of $\mathrm{O}_{3}$ and $\mathrm{PM}_{2.5}$ in rural India are relatively scarce, it has become clear that household combustion is a major contributor to ambient levels of these pollutants. For example Balakrishnan et al. (2013) measured $\mathrm{PM}_{2.5}$ concentrations in households and observed mean $24 \mathrm{~h}$ concentrations of $163 \mu \mathrm{g} \mathrm{m}^{-3}$ in the living room and $609 \mu \mathrm{g} \mathrm{m}^{-3}$ in the kitchen. Over the last half decade, several researchers have, through independent studies, come to the conclusion that a significant fraction $(22 \%-52 \%)$ of ambient $\mathrm{PM}_{2.5}$ is directly emitted from residential cooking and heating (Butt et al., 2016; Chafe et al., 2014; Conibear et al., 2018; GBD MAPS Working Group, 2018; Guttikunda et al., 2016; Klimont et al., 2017; Lelieveld et al., 2015; Silva et al., 2016).

Residences in India were estimated to consume 220, 86.5, and $93.0 \mathrm{Tg} \mathrm{yr}^{-1}$ of dry matter of wood fuel, agricultural residues, and dung, respectively, in the year 1985 (Yevich and Logan, 2003). While the fraction of Indians using biomass cook fuels is decreasing, the total population is increasing such that biomass fuels are still being utilized at approximately the same overall level (Pandey et al., 2014). Emissions of primary $\mathrm{PM}_{2.5}$ from residential cooking in India were estimated to be $2.6 \mathrm{Tg} \mathrm{yr}^{-1}$ based on a compiled emissions inventory (Pandey et al., 2014). Additionally, Pandey et al. (2014) estimated that $4.9 \mathrm{Tg}$ of non-methane volatile organic compounds (NMVOCs) are produced annually in India from residential cooking. This suggests that additional $\mathrm{PM}_{2.5}$ mass may be formed via secondary pathways from the oxidation of NMVOCs and either nucleation of new particles or condensation onto existing $\mathrm{PM}_{2.5}$. Alternatively, these nonmethane VOCs could contribute to photochemical ozone production in the presence of $\mathrm{NO}_{x}$ (Finlayson-Pitts and Pitts, 2000).

In this study, we quantified emissions of $\mathrm{CO}, \mathrm{CO}_{2}$, and 76 different VOCs from 55 cook fires carried out by a local cook in a village home cooking typical meals. This is a substantially updated version of the work done in simulated village houses in India and China in the 1990s, where 58 fuelstove combinations were measured in semi-controlled conditions using water boiling tests including a number of nonbiomass stoves, although a similar set of pollutants were measured (Smith et al., 2000b, a; Tsai et al., 2003; Zhang et al., 2000). This time, we measured emissions in field conditions from two traditional, locally made cookstoves, the chulha and the angithi. The former is a primarily flaming stove with generally higher modified combustion efficiencies or concentration ratios of carbon dioxide to the sum of carbon dioxide and carbon monoxide (average dung-chulha: 0.865 ), used to cook village meals. The angithi is largely smoldering with lower modified combustion efficiencies (average dung-angithi: 0.819 ) and is primarily used for cooking animal fodder and simmering milk. We measured emissions from cookstoves with two kinds of biomass: the most popular biomass type, brushwood (Census of India, 2011), and dung cakes. The fuels and stoves used in this study are predominantly used in the Indo-Gangetic Plain. Our first objective is to characterize emissions of select VOCs and $\mathrm{PM}_{2.5}$ from these fuel-stove combinations. Subsequently, with the aid of secondary organic aerosol (SOA) potential values from Derwent et al. (2010), incremental reactivities first described in Carter (1994), and second-order rate coefficients with $\mathrm{OH}$ combined with our emission factors, we estimate SOA-forming potentials, excess ozone mixing ratios in a VOC-limited regime, and $\mathrm{OH}$ reactivities, respectively. Given their widespread use in India, emissions from these biomass-burning stoves are estimated to impact regional air quality due to both primary and secondary organic aerosol and ozone formation.

\section{Experimental methods}

\subsection{Field site and sample collection}

The field office was located at the SOMAARTH Demographic, Development, and Environmental Surveillance Site in Palwal District, Haryana, India, run by the International Clinical Epidemiological Network (INCLEN). The site consists of 51 villages in the area with roughly 200000 inhabitants (Balakrishnan et al., 2015; Mukhopadhyay et al., 2012; Pillarisetti et al., 2014).

Samples were collected from cookstove emissions between 5 August and 3 September 2015. Cooking events occurred at a village kitchen in Khatela, Palwal District. A local cook was hired to prepare meals for human consumption consisting of either chapati or rice with vegetables using a chulha stove, as well as animal food using an angithi stove.

Animal fodder simmers in a pot set upon smoldering dung in a clay bowl, referred to as an angithi. Chulha stoves are made from bricks and a covering of clay, and the availability of oxygen from the packing of biomass fuels results in primarily flaming combustion. The chulha is used to cook most meals for families in this village. Buffalo and cow dung patties and brushwood, in the form of branches and twigs, were used in chulha stoves, and for the 13 mixed fuel cooking events dung and brushwood were combined in a ratio determined by the cook's preference. Stoves and food ingredients were produced and fuels procured by the household or village. Fuel moisture content, fuel mass burned, and meals cooked were noted for each cook fire and can be found in the Supplement. Additional information regarding the cooking events and setup can be found in Fleming et al. (2018).

The sampling scheme is illustrated in Fig. 1. Air sampling pumps (PCXR-8, SKC Inc.) created a flow of emissions through the sampling apparatus. Emissions were captured with three-pronged probes that were fixed $60 \mathrm{~cm}$ above the cookstove. $\mathrm{PM}_{2.5}$ emissions and gases were sampled through cyclones $(2.5 \mu \mathrm{m}$, URG Corporation). The resulting flow 


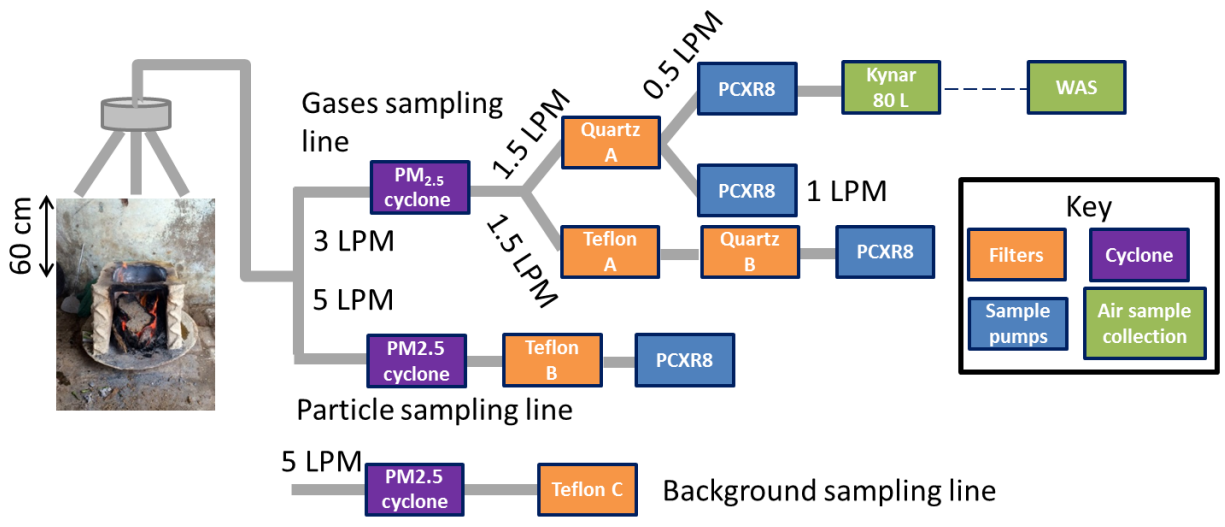

Figure 1. Sampling train for collecting cookstove emissions. PCXR8 (blue) are sampling pumps, WAS or whole-air samples (green) are the air samplers, and orange boxes are Teflon or quartz filters used to collect $\mathrm{PM}_{2.5}$.

of particles was captured on either quartz or polytetrafluoroethylene (PTFE) filters, while gases were collected in an $80 \mathrm{~L}$ Kynar bag throughout the entire cooking event. Flows were measured both before and after sampling to ensure they did not change by more than $10 \%$ using a mass flowmeter (TSI 4140). After the cooking event, pumps were turned off and a whole-air sampler, consisting of a stainless-steel canister (2 L) welded to a bellows-sealed valve (Swagelok), was filled to ambient pressure from the Kynar bag. Wholeair samplers were thoroughly flushed and evacuated in the Rowland-Blake laboratory before being shipped to India. At the end of the measurement campaign, whole-air samplers were shipped back to the laboratory and analyzed within 2 months of the end of the field measurements. A "grab" whole-air sample (WAS) was collected before cooking commenced each day. This served as a background for all cooking events sampled on that day.

\subsection{Gas chromatography analysis}

Colman et al. (2001) described the VOC analysis protocol in detail. Briefly, a known amount of the WAS flowed over glass beads inside a U-shaped trap cooled by liquid nitrogen. The flow was regulated by a mass flow controller and resulted in a known, roughly 600 Torr drop in the pressure in the whole-air sampler. High-volatility gases such as $\mathrm{O}_{2}$ and $\mathrm{N}_{2}$ passed over the beads, while lower-volatility gases adsorbed onto the beads. Compounds were re-volatilized by immersing the trap in hot water and were injected into a $\mathrm{He}$ carrier gas stream where the flow was split equally to five columns housed in three gas chromatographs (HP-6890). The compounds were separated by gas chromatography (GC) and subsequently detected by two electron capture detectors, two flame ionization detectors, and one quadrupole mass spectrometer detector. Peaks corresponding to compounds of interest were integrated manually. $\mathrm{CO}, \mathrm{CO}_{2}$, and $\mathrm{CH}_{4}$ were analyzed using two $\mathrm{GC}$ systems equipped with thermal conductivity and flame ionization detectors as described in Simpson et al. (2014). The $\mathrm{CO}$ and $\mathrm{CO}_{2} \mathrm{GC}$ with flame ionization detection (GC-FID) system is equipped with a Ni catalyst that converts $\mathrm{CO}$ into detectable $\mathrm{CH}_{4}$.

\subsection{Gas and $\mathbf{P M}_{2.5}$ emission factor calculations}

Emission factors (EFs) were calculated using the carbonbalance method, which assumes that all carbon in the fuel is converted to $\mathrm{CO}_{2}, \mathrm{CO}$, VOCs, and PM when the fuel is burned. The total gas-phase carbon emissions were approximated with the concentrations of $\mathrm{CO}_{2}, \mathrm{CO}$, and 76 detected VOCs measured using WAS. This is a good approximation since most emitted carbon resides in $\mathrm{CO}_{2}$ and $\mathrm{CO}(>95 \%)$, so the error associated with VOCs that are not detected is relatively small (Roden and Bond, 2006; Smith et al., 1993; Wathore et al., 2017; Zhang et al., 2000). The mass of carbon in species $i\left(m_{i, \mathrm{C}}\right)$ was calculated using Eq. (1):

$$
\begin{aligned}
& m_{i, \mathrm{C}}(\mathrm{g})= \\
& \frac{C_{i, \mathrm{C}}\left(\mathrm{g} \mathrm{m}^{-3}\right)}{C_{\mathrm{CO}_{2}, \mathrm{C}}+C_{\mathrm{CO}, \mathrm{C}}+C_{\mathrm{CH}_{4}, \mathrm{C}}+\ldots+C_{\mathrm{C}_{6} \mathrm{H}_{6}, \mathrm{C}}\left(\mathrm{g} \mathrm{m}^{-3}\right)} \\
& \cdot m_{\mathrm{T}, \mathrm{C}}(\mathrm{kg}) \cdot \frac{1000 \mathrm{~g}}{1 \mathrm{~kg}}
\end{aligned}
$$

where $C_{i, \mathrm{C}}$ represents the mass concentration of carbon for species $i$, and $m_{\mathrm{T}, \mathrm{C}}$ refers to the carbon mass of the fuel, adjusted for ash and char carbon. Fuels were weighed before they were burned, and the dry mass was calculated based on moisture content measurements. The ash was weighed after the cooking event and subtracted from the dry mass of the fuel giving the net dry fuel burned for the cooking event, $m_{\mathrm{T}}$. When mixtures of dung and brushwood were used, both were individually weighed to more accurately determine the carbon mass burned. The fraction of carbon in the fuel used to yield $m_{\mathrm{T}, \mathrm{C}}$ was taken to be 0.33 for buffalo and cow dung and 0.45 for brushwood fuels based on Smith et al. (2000a, b). Carbon in ash was estimated as $2.9 \%$ and $80.9 \%$ of the measured char mass for dry dung and dry brushwood, re- 
spectively (Smith et al., 2000b). For each species with $n_{\mathrm{c}, i}$ carbon atoms in the formula and molecular weight $\left(\mathrm{MW}_{i}\right)$, the emissions factor $\left(\mathrm{EF}_{i}\right)$ was calculated using Eq. (2).

$\mathrm{EF}_{i}\left(\frac{\mathrm{g} \mathrm{VOC}_{i}}{\mathrm{~kg} \text { fuel }}\right)=\frac{m_{i, \mathrm{C}}(\mathrm{g}) \cdot \frac{\mathrm{MW}_{i}\left(\mathrm{~g} \mathrm{~mol}^{-1}\right)}{n_{\mathrm{c}, i} \times 12.00\left(\mathrm{~g} \mathrm{~mol}^{-1}\right)}}{m_{\mathrm{T}}(\mathrm{kg})}$

In addition to the emission factor normalized by the total fuel mass, emission factors were normalized to the total carbon mass in the fuel, calculated via Eq. (3).

$\mathrm{EF}_{i}\left(\frac{\mathrm{g} \mathrm{VOC}_{i}}{\mathrm{~kg} \text { fuelC }}\right)=\mathrm{EF}_{i}\left(\frac{\mathrm{g} \mathrm{VOC}_{i}}{\mathrm{~kg} \text { fuel }}\right) \cdot \frac{m_{\mathrm{T}}(\mathrm{kg})}{m_{\mathrm{T}, \mathrm{C}}(\mathrm{kg})}$

$\mathrm{PM}_{2.5}$ mass was determined gravimetrically using Teflon filters (PTFE, SKC Inc., $47 \mathrm{~mm}$ ) weighed on a Cahn-28 electrobalance with a repeatability of $\pm 1.0 \mu \mathrm{g}$ after equilibrating for a minimum of $24 \mathrm{~h}$ in a humidity- and temperaturecontrolled environment both before and after sample collection (average temperature: $19.5 \pm 0.5^{\circ} \mathrm{C}$; average relative humidity: $49 \pm 5 \%$ ). Another gravimetric filter was collected in the background during the cooking event and was equilibrated and weighed in the same way (Fig. 1, Teflon C). Five field blanks filters were prepared by opening filters and then immediately closing and sealing the filters the same way as all samples; these filters had negligible mass loading (average: $0.40 \mu \mathrm{g}$ ) relative to samples (average: $1.57 \mathrm{mg}$ ). The method detection limit was determined to be $9.3 \mu \mathrm{g}$ from the standard deviation of the field blank filters multiplied by 3 . The background filter mass (Fig. 1, Teflon C) was adjusted by matching the sampling volume to that of the sample gravimetric Teflon filter (Fig. 1, Teflon A). This assumes that the mass of $\mathrm{PM}_{2.5}$ collected on the filter is directly proportional to the flow rate through the filter. The background mass was then subtracted from the sample mass to obtain the mass of $\mathrm{PM}\left(m_{\mathrm{PM}}\right)$ in Eq. (4) below.

$\frac{\mathrm{EF}_{\mathrm{PM}}}{\mathrm{EF}_{\mathrm{CO}}}=\frac{m_{\mathrm{PM}} / V_{\mathrm{air}}}{m_{\mathrm{CO}} / V_{\mathrm{air}}}$

\subsection{Modified combustion efficiency (MCE)}

Modified combustion efficiency is defined as follows:

$\mathrm{MCE}=\frac{\Delta \mathrm{CO}_{2}}{\Delta \mathrm{CO}+\Delta \mathrm{CO}_{2}}$,

where $\triangle \mathrm{CO}$ and $\Delta \mathrm{CO}_{2}$ are background-subtracted mixing ratios of $\mathrm{CO}$ and $\mathrm{CO}_{2}$ for the time-integrated (WAS). The total carbon mixing ratio is approximated by the sum of carbon monoxide and carbon dioxide in this definition.

\subsection{SOA-forming potential}

Relative SOA-forming potential from measured VOCs was estimated using secondary organic aerosol potential (SOAP) values from Derwent et al. (2010), who used a photochemical transport model to simulate the SOA mass increase from the instantaneous emission of a particular VOC in a single parcel of air traveling across Europe. The model was outfitted with the Master Chemical Mechanism (MCM v.3.1) and the UK National Atmospheric Emission Inventory. The model was initialized with 2 ppbv $\mathrm{NO}$ and 6 ppbv $\mathrm{NO}_{2}$ (Derwent et al., 1998). SOA mass was estimated assuming equilibrium partitioning of oxidation products. Partitioning coefficients were calculated using absorptive partitioning theory of Pankow (1994). In the SOAP approach, all SOA mass increases from a particular VOC $(i)$ are normalized to that of toluene as shown in Eq. (6).

$\operatorname{SOAP}_{i}=$

increment in SOA mass concentration with species $i$

increment in SOA mass concentration with toluene $\times 100$

SOA-forming potential was calculated from the published SOAP values using Eq. (7).

SOA potential $=\sum_{i=0}^{n} \mathrm{SOAP}_{i} \times \mathrm{EF}_{i}\left(\frac{\mathrm{g} \mathrm{VOC}_{i}}{\mathrm{kgfuelC}_{\mathrm{C}}}\right)$

Table S1 in the Supplement lists the SOAP values used to calculate SOA-forming potential in this study. We emphasize that the SOA-forming potential presented here is a relative value and does not represent an absolute SOA yield.

\subsection{OH reactivity}

Total $\mathrm{OH}$ reactivity normalized by the mixing ratio of $\mathrm{CO}$ was calculated using Eq. (8).

$$
\begin{gathered}
\text { OH reactivity }\left(\frac{1}{\text { sppvcO }^{2}}\right)=\sum_{i=0}^{n} k_{\mathrm{OH}, i}\left(\frac{\mathrm{cm}^{3}}{\text { molec s}}\right) \\
\times \mathrm{ER}_{i}\left(\frac{\operatorname{pptv} \mathrm{VOC}_{i}}{\operatorname{ppbvCO}}\right) \times 2.46 \times 10^{7}\left(\frac{\mathrm{molec}}{\mathrm{cm}^{3} \mathrm{pptv}}\right)
\end{gathered}
$$

Second-order rate constants $\left(k_{\mathrm{OH}}\right)$ at $25^{\circ} \mathrm{C}$ were taken from the NIST chemical kinetics database (Manion et al., 2015). Table $\mathrm{S} 1$ reproduces the $k_{\mathrm{OH}}$ constants used in the study. $\mathrm{ER}_{i}$ is the emission ratio for compound $i$ in pptv of VOC per ppbv of $\mathrm{CO}$. The last term serves as a conversion factor from VOC mixing ratio to concentration at standard ambient temperature and pressure $\left(25^{\circ} \mathrm{C}, 1 \mathrm{~atm}\right)$. By using the emission ratio to $\mathrm{CO}$, we can track $\mathrm{OH}$ reactivity from VOCs depending on the extent of dilution from the plume. From here forward, the $\mathrm{OH}$ reactivity (per second) reported is the average at the location of the sampling probes, or roughly $60 \mathrm{~cm}$ above the cookstove.

\subsection{Ozone-forming potential (OFP)}

The ozone-forming potential was estimated from the incremental reactivity of VOCs tabulated in Carter (2010). Incremental reactivities were calculated by comparing ozone 
formation before and after a VOC was introduced in a box model simulation. The maximum incremental reactivity (MIR) scenario sets high $\mathrm{NO}_{x}$ concentrations, optimized to yield the largest incremental ozone production. In other words, ozone production was VOC limited. Because of this, the OFPs given here represent a scenario where VOC emissions from cooking have the largest impact on ozone production. This high- $\mathrm{NO}_{x}$ scenario was chosen for high sensitivity in ozone production from cooking emission VOCs. In addition, it is expected to be more realistic for a smoke plume in which $\mathrm{NO}_{x}$ is co-emitted with VOCs. OFPs were calculated using Eq. (9).

$$
\begin{aligned}
& \operatorname{OFP}\left(\frac{\mathrm{gO}_{3}}{\mathrm{~kg} \text { fuelC }}\right)=\sum_{i=0}^{n} \operatorname{MIR}\left(\frac{\mathrm{g} \mathrm{O}_{3}}{\mathrm{~g} \mathrm{VOC}_{i}}\right) \\
& \quad \times \mathrm{EF}_{i}\left(\frac{\mathrm{g} \mathrm{VOC}_{\mathrm{i}}}{\mathrm{kgfuelC}_{\text {fuel }}}\right)
\end{aligned}
$$

OFPs used in this study are listed next to the corresponding compound in Table $\mathrm{S} 1$.

\subsection{Statistical analysis}

One-way analysis of variance (ANOVA) with Tukey post hoc testing was utilized to determine if there were significant differences $(p<0.05)$ in emissions of specific VOCs among categorical variables, i.e., stove and fuel types. All analyses were performed in R version 3.4.0 and RStudio version 1.0.143 (RStudio Team, 2016).

\section{Results and discussion}

\subsection{Chemical composition}

Average VOC and $\mathrm{PM}_{2.5}$ EFs ( $\mathrm{g} \mathrm{kg}^{-1}$ dry fuel) as well as MCE are given in Table 1. The compounds are grouped by fuel-stove combination, with major species $\left(\mathrm{CO}_{2}, \mathrm{CO}\right.$, $\mathrm{CH}_{4}$, and $\mathrm{PM}_{2.5}$ ) listed first, followed by sulfur-containing compounds, halogen-containing compounds, organonitrates, alkanes, alkenes, alkynes, aromatics, terpenes, and oxygenated compounds. The sample size $(n)$ used for calculating the average values and standard deviations was $n=18$ for dung-chulha, $n=14$ for brushwood-chulha, $n=13$ for mixed fuel-chulha, and $n=10$ for dung-angithi. For the majority of the compounds, the standard deviations are smaller than or comparable to the average values, indicating fair reproducibility. There are many factors that may lead to variability in biomass burning emissions, including pyrolysis temperature (Chen and Bond, 2010), fuel moisture content (Tihay-Felicelli et al., 2017), and the wind speed/direction (Surawski et al., 2015), among others. Relationships between emissions and fuel moisture content (Fig. S1) or meal cooked were not found to be significant for any compounds (all $p<0.05$ ). This paper therefore focuses on the relationships between emissions and fuel-stove combination.
Figure $2 \mathrm{a}$ visually shows the mass fraction attributed to each compound class for the measured gas-phase emissions. The total EFs given below the pie charts are normalized by fuel carbon in Fig. 2a in order to compare between cook fires generated with dung, wood, and wood-dung mixtures, which have different carbon contents. The total measured VOC emissions from dung-angithi were roughly twice those of dung-chulha in terms of gram per kilogram fuel carbon. Further, dung-chulha emitted more than twice the amount emitted by brushwood-chulha. The most prominent difference is non-furan oxygenates, making up almost half of all brushwood-chulha emissions and a smaller fraction for other fuel-stove combinations. While oxygenates make up a higher fraction of brushwood-chulha emissions, the absolute EFs for oxygenates from dung-burning and angithi cook fires are higher as discussed later in more detail.

Table 2 shows EFs $\left(\mathrm{g} \mathrm{kg}^{-1}\right.$ fuel C) for select VOCs. The differences in mean EFs for each fuel-stove combination are also included in Table 2. Mean differences in EFs reported for chulha and angithi stoves were calculated for cook fires utilizing only dung fuels. Likewise, mean EFs for wood and dung cook fires only represent cooking events using the chulha. This was done to isolate a single variable - either fuel or stove type. For all alkanes and most alkenes, we measured higher emissions for dung-angithi cook fires (Table 2). Also, from the mean differences in EFs, we found that stovespecific combustion conditions impact emissions more than the selection of fuel type. The difference is so dramatic for alkanes and most alkenes that the mean difference in EFs for cookstoves burning dung is always larger than the mean $\mathrm{EF}$ of that compound. For comparison, the mean difference in EFs for chulha cookstoves is always lower than the overall mean EF. Ethene was an exception; there was no relationship between ethene emissions and stove type. On the other hand, the mean EF of ethene by dung cook fires was very large compared to mean EFs from brushwood cook fires, with a mean difference in EFs of $4.05 \mathrm{~g} \mathrm{~kg}^{-1}$ fuel C. Some alkenes with two double bonds were also exceptions. For 1,3-butadiene ( $p=0.06)$ and 1,2-butadiene ( $p=0.089$ ), stove and EF may or may not have a significant relationship. 1,2-Propadiene emissions from chulha cookstoves are higher $(p<0.01)$. All three compounds still show a significant relationship to fuel type, with EFs being higher for dung cook fires.

Similar to alkanes and alkenes, aromatics, oxygenates, and halogen- and sulfur-containing compounds all had higher emissions per kilogram of fuel carbon when dung fuels and angithi stoves were utilized compared to brushwood fuels and chulha stoves, respectively. We focus on the behavior of the most interesting groups of compounds in the discussion below.

The chlorine-containing organic compounds are generally not expected to come from cook fires in large quantities. However, we observed an interesting practice in which the cook often used plastic bags to start the fire, which could be 
Table 1. Averaged emission factors and standard deviation of $\mathrm{PM}_{2.5}$ and gas-phase species ( $\mathrm{g} \mathrm{kg}^{-1} \mathrm{dry}$ fuel) for dung-chulha, brushwoodchulha, mixed fuel-chulha, and dung-angithi cook fires. Previously published emission factors ( $\mathrm{g} \mathrm{kg}^{-1}$ dry fuel) from dung and hardwood cook fires are shown for comparison (Stockwell et al., 2016). Sample sizes for the current study ( $n$ ) were $n=18$ for dung-chulha, $n=14$ for brushwood-chulha, $n=13$ for mixed-chulha, and $n=10$ for dung-angithi.

\begin{tabular}{|c|c|c|c|c|c|c|}
\hline $\begin{array}{l}\text { Compound } \\
\text { (formula) }\end{array}$ & $\begin{array}{l}\text { Dung-chulha } \\
\text { average (SD) }\end{array}$ & $\begin{array}{r}\text { Brushwood-chulha } \\
\text { average (SD) }\end{array}$ & $\begin{array}{r}\text { Mixed fuel-chulha } \\
\text { average (SD) }\end{array}$ & $\begin{array}{l}\text { Dung-angithi } \\
\text { average (SD) }\end{array}$ & $\begin{array}{r}\text { Stockwell et al. (2016) } \\
\text { dung } \\
\text { average (SD) }\end{array}$ & $\begin{array}{r}\text { Stockwell et al. (2016) } \\
\text { hardwood } \\
\text { average (SD) }\end{array}$ \\
\hline Modified combustion & $0.865(0.014)$ & $0.937(0.035)$ & $0.892(0.021)$ & $0.819(0.031)$ & 0.898 & 0.923 \\
\hline Efficiency & & & & & & \\
\hline $\mathrm{PM}_{2.5}$ & $19.2(7.1)$ & $7.42(5.67)$ & $11.0(2.0)$ & $33.2(7.6)$ & $14.73(0.33)^{\mathrm{a}}$ & $7.97(3.80)^{\mathrm{a}}$ \\
\hline Carbon dioxide $\left(\mathrm{CO}_{2}\right)$ & $984(23)$ & $1242(61)$ & $969(31)$ & $888(48)$ & $1129(80)$ & $1462(16)$ \\
\hline Carbon monoxide (CO) & $97.7(9.5)$ & $53.0(30.1)$ & $74.8(16.0)$ & $125(20)$ & $80.9(13.8)$ & $77.2(13.5)$ \\
\hline Methane $\left(\mathrm{CH}_{4}\right)$ & $6.92(1.23)$ & $4.80(2.09)$ & $4.84(0.89)$ & $15.1(2.6)$ & $6.65(0.46)$ & $5.16(1.39)$ \\
\hline \multicolumn{7}{|l|}{ Sulfur-containing } \\
\hline Carbonyl sulfide (OCS) & $0.124(0.040)$ & $1.44(0.54) \times 10^{-2}$ & $8.50(2.42) \times 10^{-2}$ & $0.352(0.217)$ & $0.148(0.123)$ & $1.87(1.15) \times 10^{-2}$ \\
\hline $\operatorname{DMS}\left(\mathrm{C}_{2} \mathrm{H}_{6} \mathrm{~S}\right)$ & $9.69(4.54) \times 10^{-3}$ & $1.39(1.34) \times 10^{-3}$ & $4.81(2.26) \times 10^{-3}$ & $4.34(3.11) \times 10^{-2}$ & $2.37(0.08) \times 10^{-2}$ & $0.255(0.359)$ \\
\hline \multicolumn{7}{|l|}{ Halogen-containing } \\
\hline $\begin{array}{l}\text { Dichloromethane } \\
\left(\mathrm{CH}_{2} \mathrm{Cl}_{2}\right)\end{array}$ & $4.46(3.94) \times 10^{-4}$ & $2.18(3.13) \times 10^{-4}$ & $4.04(6.44) \times 10^{-4}$ & $4.56(2.73) \times 10^{-4}$ & $\mathrm{~nm}^{\mathrm{b}}$ & $\mathrm{nm}$ \\
\hline Chloromethane $\left(\mathrm{CH}_{3} \mathrm{Cl}\right)$ & $1.78(0.70)$ & $0.280(0.157)$ & $1.02(0.42)$ & $4.58(1.89)$ & $1.60(1.53)$ & $2.36(1.62) \times 10^{-2}$ \\
\hline Bromomethane $\left(\mathrm{CH}_{3} \mathrm{Br}\right)$ & $6.57(2.78) \times 10^{-3}$ & $7.92(2.13) \times 10^{-4}$ & $4.35(1.81) \times 10^{-3}$ & $1.43(0.57) \times 10^{-2}$ & $5.34(3.02) \times 10^{-3}$ & $5.61(3.01) \times 10^{-4}$ \\
\hline Iodomethane $\left(\mathrm{CH}_{3} \mathrm{I}\right)$ & $6.10(4.78) \times 10^{-4}$ & $9.62(2.31) \times 10^{-5}$ & $2.41(0.66) \times 10^{-4}$ & $8.83(1.62) \times 10^{-4}$ & $4.39(1.78) \times 10^{-4}$ & $1.23(1.11) \times 10^{-4}$ \\
\hline Ethyl chloride $\left(\mathrm{C}_{2} \mathrm{H}_{5} \mathrm{Cl}\right)$ & $2.54(1.17) \times 10^{-3}$ & $4.22(3.72) \times 10^{-4}$ & $1.59(0.67) \times 10^{-3}$ & $9.11(3.50) \times 10^{-3}$ & $\mathrm{~nm}$ & \\
\hline $\begin{array}{l}\text { Dichloroethane } \\
\left(\mathrm{C}_{2} \mathrm{H}_{4} \mathrm{Cl}_{2}\right)\end{array}$ & $8.80(2.98) \times 10^{-4}$ & $2.55(2.17) \times 10^{-4}$ & $1.21(2.32) \times 10^{-3}$ & $1.47(0.91) \times 10^{-3}$ & $4.97 \times 10^{-3 c}$ & $1.24(0.30) \times 10^{-4}$ \\
\hline \multicolumn{7}{|l|}{ Nitrates } \\
\hline $\begin{array}{l}\text { Methyl nitrate } \\
\left(\mathrm{CH}_{3} \mathrm{ONO}_{2}\right)\end{array}$ & $1.83(5.18) \times 10^{-3}$ & $5.34(14.4) \times 10^{-3}$ & $6.60(11.7) \times 10^{-3}$ & $0.170(0.339)$ & $1.46(1.94) \times 10^{-2}$ & $6.96(5.73) \times 10^{-3}$ \\
\hline Ethyl nitrate $\left(\mathrm{CH}_{3} \mathrm{ONO}_{2}\right)$ & $2.37(3.86) \times 10^{-4}$ & $5.54(10.2) \times 10^{-4}$ & $2.27(6.40) \times 10^{-3}$ & $4.53(11.6) \times 10^{-2}$ & $\mathrm{~nm}$ & $\mathrm{~nm}$ \\
\hline $\begin{array}{ll}i \text {-Propyl } & \text { nitrate } \\
\left(\mathrm{C}_{3} \mathrm{H}_{7} \mathrm{ONO}_{2}\right) & \end{array}$ & $1.90(1.61) \times 10^{-4}$ & $2.40(4.92) \times 10^{-4}$ & $4.10(8.38) \times 10^{-4}$ & $5.90(12.1) \times 10^{-3}$ & $\mathrm{~nm}$ & $\mathrm{~nm}$ \\
\hline $\begin{array}{l}n \text {-Propyl } \\
\left(\mathrm{C}_{3} \mathrm{H}_{7} \mathrm{ONO}_{2}\right)\end{array}$ & $6.32(5.23) \times 10^{-5}$ & $9.01(14.1) \times 10^{-5}$ & $1.44(3.25) \times 10^{-4}$ & $1.82(4.35) \times 10^{-3}$ & $\mathrm{~nm}$ & $\mathrm{~nm}$ \\
\hline $\begin{array}{l}\text { 2-Butyl nitrate } \\
\left(\mathrm{C}_{4} \mathrm{H}_{9} \mathrm{ONO}_{2}\right) \quad \text { }\end{array}$ & $2.69(2.14) \times 10^{-4}$ & $1.05(1.13) \times 10^{-4}$ & $7.10(20.3) \times 10^{-4}$ & $2.45(4.09) \times 10^{-3}$ & $\mathrm{~nm}$ & $\mathrm{~nm}$ \\
\hline $\begin{array}{l}\text { 3-Pentyl nitrate } \\
\left(\mathrm{C}_{5} \mathrm{H}_{11} \mathrm{ONO}_{2}\right) \quad\end{array}$ & $4.75(1.61) \times 10^{-5}$ & $2.29(2.08) \times 10^{-5}$ & $3.13(1.97) \times 10^{-5}$ & $1.94(4.06) \times 10^{-4}$ & $\mathrm{~nm}$ & $\mathrm{~nm}$ \\
\hline $\begin{array}{l}\text { 2-Pentyl nitrate } \\
\left(\mathrm{C}_{5} \mathrm{H}_{11} \mathrm{ONO}_{2}\right)\end{array}$ & $2.37(2.10) \times 10^{-5}$ & $1.63(2.46) \times 10^{-5}$ & $1.25(1.26) \times 10^{-5}$ & $1.82(4.54) \times 10^{-4}$ & $\mathrm{~nm}$ & $\mathrm{~nm}$ \\
\hline \multicolumn{7}{|l|}{ Alkanes } \\
\hline Ethane $\left(\mathrm{C}_{2} \mathrm{H}_{6}\right)$ & $0.717(0.193)$ & $0.380(0.247)$ & $0.422(0.096)$ & $2.06(0.69)$ & $1.08(0.30)$ & $0.160(0.122)$ \\
\hline Propane $\left(\mathrm{C}_{3} \mathrm{H}_{8}\right)$ & $0.211(0.073)$ & $9.48(8.41) \times 10^{-2}$ & $0.116(0.032)$ & $0.819(0.157)$ & $0.457(0.137)$ & $0.202(0.140)$ \\
\hline$i$-Butane $\left(\mathrm{C}_{4} \mathrm{H}_{10}\right)$ & $1.73(0.71) \times 10^{-2}$ & $4.60(4.86) \times 10^{-3}$ & $9.51(2.75) \times 10^{-3}$ & $7.27(1.54) \times 10^{-2}$ & $0.215(0.126)$ & $0.406(0.478)$ \\
\hline$n$-Butane $\left(\mathrm{C}_{4} \mathrm{H}_{10}\right)$ & $4.71(1.88) \times 10^{-2}$ & $1.57(1.67) \times 10^{-2}$ & $2.68(0.88) \times 10^{-2}$ & $0.215(0.047)$ & $0.29(0.09)$ & $1.11(1.48)$ \\
\hline$n$-Pentane $\left(\mathrm{C}_{5} \mathrm{H}_{12}\right)$ & $2.01(0.98) \times 10^{-2}$ & $4.44(4.08) \times 10^{-3}$ & $9.12(3.71) \times 10^{-3}$ & $6.80(2.95) \times 10^{-2}$ & $0.190(0.254)$ & $2.18(1.73) \times 10^{-2}$ \\
\hline$n$-Hexane $\left(\mathrm{C}_{6} \mathrm{H}_{14}\right)$ & $1.03(0.47) \times 10^{-2}$ & $1.96(1.58) \times 10^{-3}$ & $5.31(1.87) \times 10^{-3}$ & $4.93(1.10) \times 10^{-2}$ & $0.291(0.248)$ & $1.85 \times 10^{-2 c}$ \\
\hline$n$-Heptane $\left(\mathrm{C}_{7} \mathrm{H}_{16}\right)$ & $7.21(3.43) \times 10^{-3}$ & $9.23(6.94) \times 10^{-4}$ & $3.92(1.23) \times 10^{-3}$ & $3.17(0.85) \times 10^{-2}$ & $0.114(0.069)$ & $1.01(1.35) \times 10^{-2}$ \\
\hline $\begin{array}{l}\text { 2-Methylpentane } \\
\left(\mathrm{C}_{6} \mathrm{H}_{14}\right)\end{array}$ & $6.21(2.81) \times 10^{-3}$ & $1.23(0.99) \times 10^{-3}$ & $2.57(1.61) \times 10^{-3}$ & $2.29(1.67) \times 10^{-2}$ & $0.231(0.192)$ & $9.93(12.9) \times 10^{-3}$ \\
\hline $\begin{array}{l}\text { 3-Methylpentane } \\
\left(\mathrm{C}_{6} \mathrm{H}_{14}\right)\end{array}$ & $3.71(1.70) \times 10^{-3}$ & $1.21(1.01) \times 10^{-3}$ & $1.57(0.76) \times 10^{-3}$ & $7.54(4.30) \times 10^{-3}$ & $0.155(0.137)$ & $6.79(6.63) \times 10^{-3}$ \\
\hline \multicolumn{7}{|l|}{ Alkenes } \\
\hline Ethene $\left(\mathrm{C}_{2} \mathrm{H}_{4}\right)$ & $1.86(0.48)$ & $0.626(0.284)$ & $1.13(0.38)$ & $1.77(0.35)$ & $4.23(1.39)$ & $2.70(1.17)$ \\
\hline Propene $\left(\mathrm{C}_{3} \mathrm{H}_{6}\right)$ & $0.807(0.235)$ & $0.286(0.202)$ & $0.417(0.091)$ & $1.61(0.33)$ & $1.47(0.58)$ & $0.576(0.195)$ \\
\hline 1-Butene $\left(\mathrm{C}_{4} \mathrm{H}_{8}\right)$ & $0.158(0.047)$ & $6.32(4.59) \times 10^{-2}$ & $8.38(1.83) \times 10^{-2}$ & $0.366(0.096)$ & $0.399(0.331)$ & $0.726(0.904)$ \\
\hline$i$-Butene $\left(\mathrm{C}_{4} \mathrm{H}_{8}\right)$ & $0.133(0.057)$ & $3.46(2.50) \times 10^{-2}$ & $6.40(1.86) \times 10^{-2}$ & $0.353(0.158)$ & $0.281(0.091)$ & $0.846(1.113)$ \\
\hline trans-2-Butene $\left(\mathrm{C}_{4} \mathrm{H}_{8}\right)$ & $4.45(1.60) \times 10^{-2}$ & $2.00(1.27) \times 10^{-2}$ & $2.38(0.70) \times 10^{-2}$ & $0.151(0.055)$ & $0.151(0.010)$ & $6.78(5.98) \times 10^{-2}$ \\
\hline cis-2-Butene $\left(\mathrm{C}_{4} \mathrm{H}_{8}\right)$ & $3.38(1.19) \times 10^{-2}$ & $1.51(0.95) \times 10^{-2}$ & $1.80(0.52) \times 10^{-2}$ & $0.107(0.047)$ & $0.102(0.016)$ & $5.51(4.76) \times 10^{-2}$ \\
\hline $\begin{array}{l}\text { 3-Methyl-1-butene } \\
\left(\mathrm{C}_{5} \mathrm{H}_{10}\right)\end{array}$ & $1.46(0.48) \times 10^{-2}$ & $5.74(4.49) \times 10^{-3}$ & $7.30(1.94) \times 10^{-3}$ & $3.82(0.88) \times 10^{-2}$ & $5.58(3.50) \times 10^{-2}$ & $7.43(5.79) \times 10^{-3}$ \\
\hline $\begin{array}{l}\text { 2-Methyl-1-butene } \\
\left(\mathrm{C}_{5} \mathrm{H}_{10}\right)\end{array}$ & $2.71(1.28) \times 10^{-2}$ & $9.96(10.9) \times 10^{-3}$ & $1.19(0.42) \times 10^{-2}$ & $7.70(3.99) \times 10^{-2}$ & $\mathrm{~nm}$ & $\mathrm{~nm}$ \\
\hline $\begin{array}{l}\text { 2-Methyl-2-butene } \\
\left(\mathrm{C}_{5} \mathrm{H}_{10}\right)\end{array}$ & $2.51(1.26) \times 10^{-2}$ & $6.40(4.78) \times 10^{-3}$ & $1.10(0.47) \times 10^{-2}$ & $9.17(4.70) \times 10^{-2}$ & $\mathrm{~nm}$ & $\mathrm{~nm}$ \\
\hline
\end{tabular}


Table 1. Continued.

\begin{tabular}{|c|c|c|c|c|c|c|}
\hline $\begin{array}{l}\text { Compound } \\
\text { (formula) }\end{array}$ & $\begin{array}{l}\text { Dung-chulha } \\
\text { average (SD) }\end{array}$ & $\begin{array}{r}\text { Brushwood-chulha } \\
\text { average (SD) }\end{array}$ & $\begin{array}{r}\text { Mixed fuel-chulha } \\
\text { average (SD) }\end{array}$ & $\begin{array}{r}\text { Dung-angithi } \\
\text { average (SD) }\end{array}$ & $\begin{array}{r}\text { Stockwell et al. (2016) } \\
\text { dung } \\
\text { average (SD) }\end{array}$ & $\begin{array}{r}\text { Stockwell et al. (2016) } \\
\text { hardwood } \\
\text { average (SD) }\end{array}$ \\
\hline 1-Pentene $\left(\mathrm{C}_{5} \mathrm{H}_{10}\right)$ & $4.17(1.59) \times 10^{-2}$ & $9.65(6.55) \times 10^{-3}$ & $2.13(0.60) \times 10^{-2}$ & $0.122(0.033)$ & $0.168(0.086)$ & $1.43(0.94) \times 10^{-2}$ \\
\hline trans-2-Pentene $\left(\mathrm{C}_{5} \mathrm{H}_{10}\right)$ & $1.74(0.65) \times 10^{-2}$ & $8.89(5.77) \times 10^{-3}$ & $8.69(2.22) \times 10^{-3}$ & $5.14(2.70) \times 10^{-2}$ & $0.115(0.035)$ & $1.05(0.83) \times 10^{-2}$ \\
\hline cis-2-Pentene $\left(\mathrm{C}_{5} \mathrm{H}_{10}\right)$ & $1.00(0.36) \times 10^{-2}$ & $5.55(3.62) \times 10^{-3}$ & $4.98(1.26) \times 10^{-3}$ & $2.50(1.28) \times 10^{-2}$ & $5.14(0.76) \times 10^{-2}$ & $8.69 \times 10^{-3 c}$ \\
\hline 1-Hexene $\left(\mathrm{C}_{6} \mathrm{H}_{12}\right)$ & $6.10(2.46) \times 10^{-2}$ & $1.26(0.73) \times 10^{-2}$ & $3.09(0.91) \times 10^{-2}$ & $0.167(0.050)$ & $\mathrm{nm}$ & $\mathrm{nm}$ \\
\hline 1,2-Propadiene $\left(\mathrm{C}_{3} \mathrm{H}_{4}\right)$ & $3.76(1.69) \times 10^{-2}$ & $1.31(0.62) \times 10^{-2}$ & $2.32(0.86) \times 10^{-2}$ & $1.80(0.923) \times 10^{-2}$ & $7.15(6.76) \times 10^{-2}$ & $2.33(1.07) \times 10^{-2}$ \\
\hline 1,2-Butadiene $\left(\mathrm{C}_{4} \mathrm{H}_{6}\right)$ & $5.54(1.68) \times 10^{-3}$ & $2.82(1.81) \times 10^{-3}$ & $3.10(1.06) \times 10^{-3}$ & $4.33(1.59) \times 10^{-3}$ & $\mathrm{~nm}$ & $\mathrm{~nm}$ \\
\hline 1,3-Butadiene $\left(\mathrm{C}_{4} \mathrm{H}_{6}\right)$ & $0.203(0.071)$ & $7.44(3.99) \times 10^{-2}$ & $0.108(0.061)$ & $0.263(0.082)$ & $0.409(0.306)$ & $0.204(0.144)$ \\
\hline Isoprene $\left(\mathrm{C}_{5} \mathrm{H}_{8}\right)$ & $8.94(5.80) \times 10^{-2}$ & $1.98(1.48) \times 10^{-2}$ & $3.03(2.39) \times 10^{-2}$ & $0.188(0.143)$ & $0.325(0.443)$ & $4.16(2.23) \times 10^{-2}$ \\
\hline 1,3-Pentadiene $\left(\mathrm{C}_{5} \mathrm{H}_{8}\right)$ & $1.96(1.05) \times 10^{-2}$ & $9.17(4.79) \times 10^{-3}$ & $9.39(6.43) \times 10^{-3}$ & $5.66(2.94) \times 10^{-2}$ & $\mathrm{~nm}$ & $\mathrm{~nm}$ \\
\hline \multicolumn{7}{|l|}{ Alkynes } \\
\hline Ethyne & $1.13(0.42)$ & $0.467(0.160)$ & $0.890(0.323)$ & $0.325(0.238)$ & $0.593(0.443)$ & $0.764(0.363)$ \\
\hline 1-Propyne & $9.42(3.46) \times 10^{-2}$ & $3.82(1.76) \times 10^{-2}$ & $5.99(2.22) \times 10^{-2}$ & $5.20(2.83) \times 10^{-2}$ & $\mathrm{~nm}$ & $\mathrm{~nm}$ \\
\hline 1-Buten-3-yne $\left(\mathrm{C}_{4} \mathrm{H}_{4}\right)$ & $5.04(1.72) \times 10^{-2}$ & $1.86(0.90) \times 10^{-2}$ & $3.46(1.53) \times 10^{-2}$ & $1.74(1.26) \times 10^{-2}$ & $\mathrm{~nm}$ & $\mathrm{~nm}$ \\
\hline 1-Butyne $\left(\mathrm{C}_{4} \mathrm{H}_{6}\right)$ & $7.72(2.29) \times 10^{-3}$ & $4.07(2.24) \times 10^{-3}$ & $4.48(1.41) \times 10^{-3}$ & $5.97(1.93) \times 10^{-3}$ & $2.29(1.38) \times 10^{-2}$ & $1.28(0.47) \times 10^{-2}$ \\
\hline 2-Butyne $\left(\mathrm{C}_{4} \mathrm{H}_{6}\right)$ & $4.31(1.15) \times 10^{-3}$ & $2.55(1.44) \times 10^{-3}$ & $2.47(0.70) \times 10^{-3}$ & $4.52(1.40) \times 10^{-3}$ & $1.86(0.91) \times 10^{-2}$ & $1.02(0.66) \times 10^{-2}$ \\
\hline 1,3-Butadyne $\left(\mathrm{C}_{4} \mathrm{H}_{2}\right)$ & $6.07(2.66) \times 10^{-3}$ & $2.71(1.21) \times 10^{-3}$ & $5.43(2.01) \times 10^{-3}$ & $1.53(1.31) \times 10^{-3}$ & $\mathrm{~nm}$ & $\mathrm{~nm}$ \\
\hline \multicolumn{7}{|l|}{ Aromatics } \\
\hline Benzene $\left(\mathrm{C}_{6} \mathrm{H}_{6}\right)$ & $1.03(0.33)$ & $0.373(0.149)$ & $0.723(0.218)$ & $0.769(0.175)$ & $1.96(0.45)$ & $1.05(0.19)$ \\
\hline Toluene $\left(\mathrm{C}_{7} \mathrm{H}_{8}\right)$ & $0.483(0.273)$ & $0.221(0.085)$ & $0.297(0.077)$ & $0.860(0.167)$ & $1.26(0.05)$ & $0.241(0.160)$ \\
\hline Ethylbenzene $\left(\mathrm{C}_{8} \mathrm{H}_{10}\right)$ & $3.41(0.791) \times 10^{-2}$ & $1.25(1.20) \times 10^{-2}$ & $1.97(0.40) \times 10^{-2}$ & $9.78(1.66) \times 10^{-2}$ & $0.366(0.085)$ & $4.19(4.25) \times 10^{-2}$ \\
\hline$m / p$-Xylene $\left(\mathrm{C}_{8} \mathrm{H}_{10}\right)$ & $6.36(1.26) \times 10^{-2}$ & $2.78(1.56) \times 10^{-2}$ & $4.03(0.98) \times 10^{-2}$ & $0.148(0.030)$ & $0.601(0.294)$ & $9.57(7.99) \times 10^{-2}$ \\
\hline$o$-Xylene $\left(\mathrm{C}_{8} \mathrm{H}_{10}\right)$ & $2.38(0.76) \times 10^{-2}$ & $8.37(5.78) \times 10^{-3}$ & $1.44(0.41) \times 10^{-2}$ & $7.96(1.91) \times 10^{-2}$ & $0.228(0.083)$ & $3.93(4.31) \times 10^{-2}$ \\
\hline Styrene $\left(\mathrm{C}_{8} \mathrm{H}_{8}\right)$ & $5.88(1.58) \times 10^{-2}$ & $2.28(1.50) \times 10^{-2}$ & $3.40(1.90) \times 10^{-2}$ & $8.63(5.96) \times 10^{-2}$ & $0.255(0.091)$ & $8.71(6.69) \times 10^{-2}$ \\
\hline$i$-Propylbenzene $\left(\mathrm{C}_{9} \mathrm{H}_{12}\right)$ & $2.91(0.77) \times 10^{-3}$ & $1.20(1.11) \times 10^{-3}$ & $1.69(0.45) \times 10^{-3}$ & $9.30(4.90) \times 10^{-3}$ & $1.87(1.40) \times 10^{-2}$ & $1.70(1.67) \times 10^{-2}$ \\
\hline $\begin{array}{l}n \text {-Propylbenzene } \\
\left(\mathrm{C}_{9} \mathrm{H}_{12}\right)\end{array}$ & $6.48(2.59) \times 10^{-3}$ & $1.84(1.65) \times 10^{-3}$ & $4.02(1.59) \times 10^{-3}$ & $3.95(2.69) \times 10^{-2}$ & $3.10(1.45) \times 10^{-2}$ & $1.78(1.58) \times 10^{-2}$ \\
\hline 3-Ethyltoluene $\left(\mathrm{C}_{9} \mathrm{H}_{12}\right)$ & $1.44(0.48) \times 10^{-2}$ & $5.46(4.40) \times 10^{-3}$ & $8.59(3.26) \times 10^{-3}$ & $7.14(4.13) \times 10^{-2}$ & $5.61(2.38) \times 10^{-2}$ & $2.62(0.54) \times 10^{-2}$ \\
\hline 4-Ethyltoluene $\left(\mathrm{C}_{9} \mathrm{H}_{12}\right)$ & $6.35(2.36) \times 10^{-3}$ & $2.54(1.81) \times 10^{-3}$ & $4.18(1.96) \times 10^{-3}$ & $3.71(2.30) \times 10^{-2}$ & $3.57(1.74) \times 10^{-2}$ & $2.07(1.19) \times 10^{-2}$ \\
\hline 2-Ethyltoluene $\left(\mathrm{C}_{9} \mathrm{H}_{12}\right)$ & $6.89(2.50) \times 10^{-3}$ & $2.70(1.68) \times 10^{-3}$ & $4.63(2.07) \times 10^{-3}$ & $3.76(2.69) \times 10^{-2}$ & $3.39(1.34) \times 10^{-2}$ & $2.10(1.16) \times 10^{-2}$ \\
\hline $\begin{array}{l}\text { 1,3,5-Trimethylbenzene } \\
\left(\mathrm{C}_{9} \mathrm{H}_{12}\right)\end{array}$ & $3.87(1.71) \times 10^{-3}$ & $1.63(1.22) \times 10^{-3}$ & $2.65(1.43) \times 10^{-3}$ & $2.23(1.60) \times 10^{-2}$ & $1.79(0.83) \times 10^{-2}$ & $2.14 \times 10^{-2 \mathrm{c}}$ \\
\hline $\begin{array}{l}\text { 1,2,4-Trimethylbenzene } \\
\left(\mathrm{C}_{9} \mathrm{H}_{12}\right)\end{array}$ & $1.04(0.46) \times 10^{-2}$ & $4.25(2.69) \times 10^{-3}$ & $7.52(4.28) \times 10^{-3}$ & $6.23(5.18) \times 10^{-2}$ & $3.91(1.65) \times 10^{-2}$ & $1.74(2.35) \times 10^{-2}$ \\
\hline $\begin{array}{l}\text { 1,2,3-Trimethylbenzene } \\
\left(\mathrm{C}_{9} \mathrm{H}_{12}\right)\end{array}$ & $4.76(2.59) \times 10^{-3}$ & $1.16(0.81) \times 10^{-3}$ & $3.84(2.69) \times 10^{-3}$ & $3.01(3.16) \times 10^{-2}$ & $2.34(0.43) \times 10^{-2}$ & $2.16 \times 10^{-2 c}$ \\
\hline \multicolumn{7}{|l|}{ Terpenes } \\
\hline$\alpha$-Pinene $\left(\mathrm{C}_{10} \mathrm{H}_{16}\right)$ & $8.30(5.40) \times 10^{-4}$ & $5.38(6.94) \times 10^{-4}$ & $7.82(6.32) \times 10^{-4}$ & $2.26(2.53) \times 10^{-3}$ & $0.35(0.49)$ & $2.02(2.33) \times 10^{-2}$ \\
\hline$\beta$-Pinene $\left(\mathrm{C}_{10} \mathrm{H}_{16}\right)$ & $2.27(1.49) \times 10^{-3}$ & $1.37(0.91) \times 10^{-3}$ & $2.76(3.15) \times 10^{-3}$ & $2.89(3.56) \times 10^{-3}$ & $0.471^{\mathrm{c}}$ & $4.67 \times 10^{-2 c}$ \\
\hline \multicolumn{7}{|l|}{ Oxygenates } \\
\hline Acetaldehyde $\left(\mathrm{C}_{2} \mathrm{H}_{4} \mathrm{O}\right)$ & $0.805(0.279)$ & $0.334(0.199)$ & $0.447(0.119)$ & $1.70(0.75)$ & $1.88(1.63)$ & $0.541(0.362)$ \\
\hline Butanal $\left(\mathrm{C}_{4} \mathrm{H}_{8} \mathrm{O}\right)$ & $4.28(1.50) \times 10^{-2}$ & $1.90(1.29) \times 10^{-2}$ & $2.68(1.05) \times 10^{-2}$ & $0.108(0.047)$ & $5.40(2.19) \times 10^{-2}$ & $8.28(6.27) \times 10^{-3}$ \\
\hline Acetone $\left(\mathrm{C}_{3} \mathrm{H}_{6} \mathrm{O}\right)$ & $0.705(0.219)$ & $0.365(0.226)$ & $0.416(0.108)$ & $2.05(0.52)$ & $1.63(0.38)$ & $0.524(0.256)$ \\
\hline 2-Butanone $\left(\mathrm{C}_{4} \mathrm{H}_{8} \mathrm{O}\right)$ & $0.172(0.057)$ & $8.00(6.18) \times 10^{-2}$ & $0.103(0.038)$ & $0.498(0.151)$ & $0.262(0.109)$ & $0.232(0.286)$ \\
\hline 2-Propenal $\left(\mathrm{C}_{3} \mathrm{H}_{4} \mathrm{O}\right)$ & $0.186(0.060)$ & $0.127(0.069)$ & $0.127(0.059)$ & $0.295(0.245)$ & $\mathrm{nm}$ & $\mathrm{nm}$ \\
\hline $\operatorname{MVK}\left(\mathrm{C}_{4} \mathrm{H}_{6} \mathrm{O}\right)$ & $0.129(0.040)$ & $6.59(4.56) \times 10^{-2}$ & $6.31(2.76) \times 10^{-2}$ & $0.280(0.147)$ & $\mathrm{nm}$ & $\mathrm{nm}$ \\
\hline Furan $\left(\mathrm{C}_{4} \mathrm{H}_{4} \mathrm{O}\right)$ & $0.109(0.041)$ & $5.98(3.37) \times 10^{-2}$ & $6.81(2.19) \times 10^{-2}$ & $0.379(0.093)$ & $0.534(0.209)$ & $0.241(0.024)$ \\
\hline 2-Methylfuran $\left(\mathrm{C}_{5} \mathrm{H}_{6} \mathrm{O}\right)$ & $0.117(0.051)$ & $5.92(4.77) \times 10^{-2}$ & $6.92(2.83) \times 10^{-2}$ & $0.488(0.227)$ & $\mathrm{nm}$ & $\mathrm{nm}$ \\
\hline Furfural $\left(\mathrm{C}_{5} \mathrm{H}_{4} \mathrm{O}_{2}\right)$ & $8.55(6.05) \times 10^{-2}$ & $4.28(5.51) \times 10^{-2}$ & $8.22(5.09) \times 10^{-2}$ & $0.316(0.133)$ & $\mathrm{nm}$ & $\mathrm{nm}$ \\
\hline Methanol $\left(\mathrm{CH}_{3} \mathrm{OH}\right)$ & $2.09(1.14)$ & $2.03(2.01)$ & $1.18(0.40)$ & $4.23(3.40)$ & $2.38(0.90)$ & $1.92(0.61)$ \\
\hline Ethanol $\left(\mathrm{CH}_{5} \mathrm{OH}\right)$ & $4.08(5.93) \times 10^{-2}$ & $2.18(2.00) \times 10^{-2}$ & $5.63(6.69) \times 10^{-2}$ & $7.62(9.08) \times 10^{-2}$ & $0.563(0.589)$ & $0.128(0.017)$ \\
\hline
\end{tabular}

${ }^{a}$ From Jayarathne et al. (2018) but part of same NAMaSTE study. ${ }^{b}$ m indicates the species was not measured. ${ }^{c}$ From Stockwell et al. (2016) indicates that the measurement was not above background.

a source of chlorine-containing compounds if composed of polyvinyl chloride. Carbonyl sulfide (OCS) is largely responsible for the yellow sulfur-containing fraction in Fig. 2a, and biomass burning is a well-known source of OCS in the atmosphere (Crutzen et al., 1979). Similar to other VOCs, OCS was significantly emitted in higher quantities when angithi stoves and dung fuels were utilized.

Benzene had higher emissions from chulha stoves, which had higher MCEs when cooking with dung fuels compared to angithi stoves (dung-chulha: $3.18 \mathrm{~g} \mathrm{~kg}^{-1}$ fuel $\mathrm{C}$; dung- 
(a) Gas-phase composition

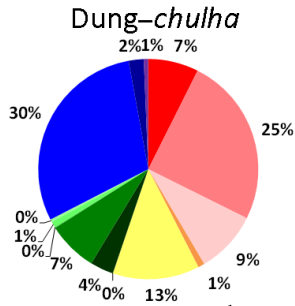

Total $=43.2 \mathrm{~g} \mathrm{VOC} \mathrm{kg}^{-1}$ fuel C

Brushwood-chulha

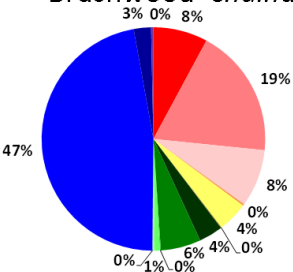

Total $=17.2 \mathrm{~g} \mathrm{VOC} \mathrm{kg}^{-1}$ fuel C

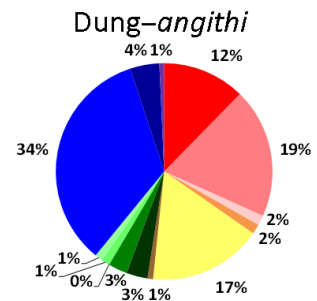

Total $=84.0 \mathrm{~g} \mathrm{VOC} \mathrm{kg}^{-1}$ fuel C

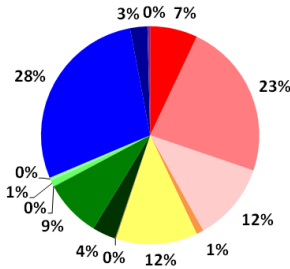

Total $=27.8 \mathrm{~g} \mathrm{VOC} \mathrm{kg}^{-1}$ fuel C Mixed-chulha

(b) $\mathrm{OH}$ reactivity

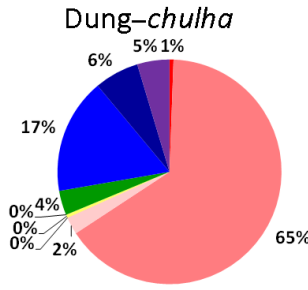

Total $=1.99 \times 10^{-2} \mathrm{~s}^{-1} \mathrm{ppb}^{-1} \mathrm{CO}$ Brushwood-chulha

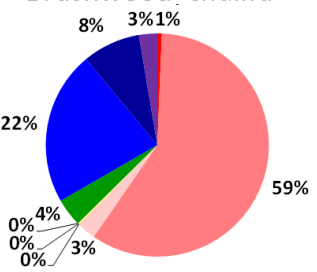

Total $=1.43 \times 10^{-2} \mathrm{~s}^{-1} \mathrm{ppb}^{-1} \mathrm{CO} \quad$ Total $=1.46 \times 10^{-2} \mathrm{~s}^{-1} \mathrm{ppb}^{-1} \mathrm{CO}$

(d) Ozone-forming potential

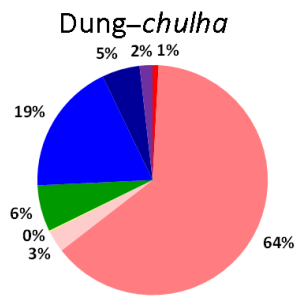

Total $=163 \mathrm{~g} \mathrm{O}_{3} \mathrm{~kg}^{-1}$ fuel $\mathrm{C}$ Brushwood-chulha
Brushwood-ch

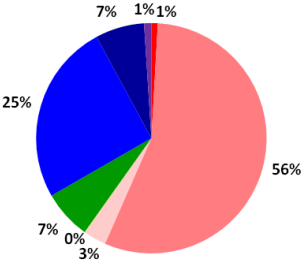

Total $=56.9 \mathrm{~g} \mathrm{O}_{3} \mathrm{~kg}^{-1}$ fuel $\mathrm{C}$

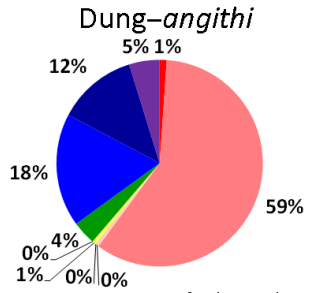

Total $=3.03 \times 10^{-2} \mathrm{~s}^{-1} \mathrm{ppb}^{-1} \mathrm{CO}$
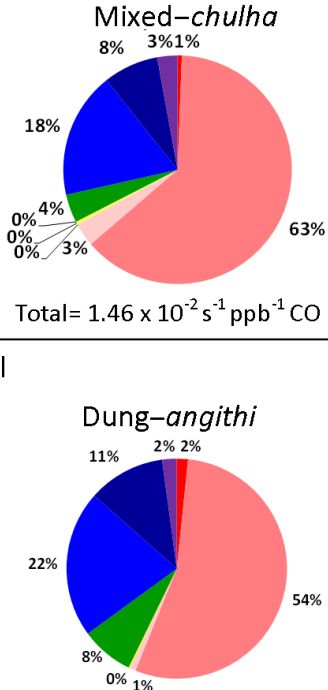

Total $=292 \mathrm{~g} \mathrm{O}_{3} \mathrm{~kg}^{-1}$ fuel C

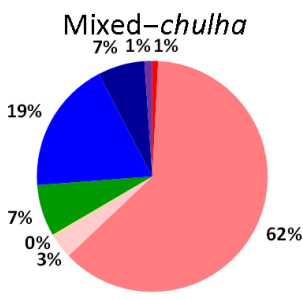

Total $=100 \mathrm{~g} \mathrm{O}_{3} \mathrm{~kg}^{-1}$ fuel C

Total $=181$ SOAP g VOC $\mathrm{kg}^{-1}$ fuel C Total $=367$ SOAP g VOC $\mathrm{kg}^{-1}$ fuel C
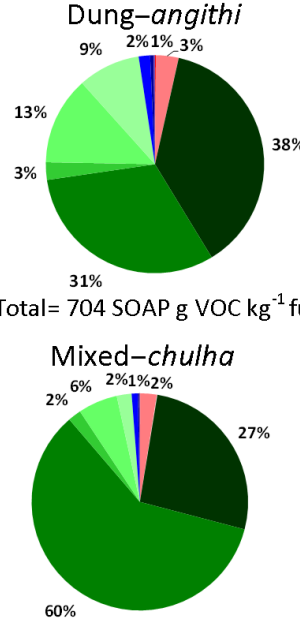

Total $=704$ SOAP g VOC $\mathrm{kg}^{-1}$ fuel C

Brushwood-chulha

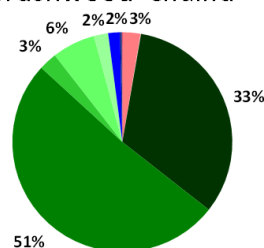

\begin{tabular}{|c|c|c|c|c|}
\hline \multicolumn{5}{|c|}{ Key } \\
\hline alkanes & Sulfur-containing & - Toluene & & D Oxygenates \\
\hline Alkenes & Halogen-containing & Benzene & & Eurans \\
\hline Alkynes & Nitrates & Styrene & $>$ Total & Terpenes \\
\hline & & $\mathrm{C}_{8}$ aromatics & aromatics & \\
\hline & & $\mathrm{C}_{9}$ aromatics & & \\
\hline
\end{tabular}

Figure 2. Pie charts showing the contribution of each species class to gas-phase composition (a), OH reactivity (b), SOAP-weighted emissions (c), and ozone-forming potential (d). For panels (b) and (d), total aromatics are shown rather than the breakdown of aromatics shown in (a) and (c). Sums of all components are shown below the pie chart. 1-Buten-3-yne is grouped in with alkynes.

angithi: $2.38 \mathrm{~g} \mathrm{~kg}^{-1}$ fuel C). As the simplest aromatic compound, benzene also had the largest average difference in fuel type EFs compared to other aromatics $\left(2.18 \mathrm{~g} \mathrm{~kg}^{-1}\right.$ fuel $\mathrm{C}$, dung-wood). This information is relevant for exposure assessment, as benzene is a known human carcinogen. Higher benzene emissions from chulha cook fires could lead to higher benzene exposures, which is a potential public health concern. However, it should be noted that the cook usually cannot control the stove used, as the angithi and chulha are used to prepare different types of meals, and exposure to benzene is not straightforward from its emission factors. 
Table 2. Emission factors ( $\mathrm{g} \mathrm{VOC} \mathrm{kg}{ }^{-1}$ fuel C) for select compounds. The mean differences between dung-angithi and dung-chulha are shown and similarly for dung-chulha and brushwood-chulha. The significance between fuel or stove and EF is indicated with asterisks. Accompanying the mean differences is the average emission factor $\left(\mathrm{g} \mathrm{VOC} \mathrm{kg}^{-1}\right.$ fuel $\mathrm{C}$ ) for dung cook fires and chulha cook fires, as well as the overall average for all performed cook fires.

\begin{tabular}{lrrrrr}
\hline Compound & $\begin{array}{r}\text { Average EF for } \\
\text { all cook fires } \\
\left(\mathrm{g} \mathrm{kg}^{-1} \text { fuel C) }\right.\end{array}$ & $\begin{array}{r}\text { Angithi-chulha } \\
\text { average EF difference } \\
\left(\mathrm{g} \mathrm{kg}^{-1} \text { fuel C) }\right.\end{array}$ & $\begin{array}{r}\text { Average EF for } \\
\text { dung fires } \\
\left(\mathrm{g} \mathrm{kg}^{-1} \text { fuel C) }\right.\end{array}$ & $\begin{array}{r}\text { Dung-brushwood } \\
\text { average EF difference } \\
\left(\mathrm{g} \mathrm{kg}^{-1} \text { fuel C }\right)\end{array}$ & $\begin{array}{r}\text { Average EF for } \\
\text { chulha cook fires } \\
\left(\mathrm{g} \mathrm{kg}^{-1} \text { fuel C) }\right.\end{array}$ \\
\hline Ethane & $2.47(2.16)$ & $4.18^{* * *}$ & $3.70(2.43)$ & $1.19^{* * *}$ & $1.60(0.744)$ \\
Propane & $0.827(0.866)$ & $1.88^{* * *}$ & $1.32(0.976)$ & $0.397^{* * *}$ & $0.448(0.256)$ \\
$n$-Butane & $0.200(0.236)$ & $0.52^{* * *}$ & $0.331(0.271)$ & $0.0568^{* * *}$ & $0.097(0.063)$ \\
\hline Ethene & $4.17(2.02)$ & $\mathrm{N} / \mathrm{A}$ & $5.64(1.32)$ & $4.05^{* * *}$ & $3.88(2.07)$ \\
Propene & $2.24(1.61)$ & $2.50^{* * *}$ & $3.38(1.48)$ & $0.213^{* * *}$ & $0.327(0.180)$ \\
1-Butene & $0.473(0.373)$ & $0.644^{* * *}$ & $0.718(0.377)$ & $2.21^{* * *}$ & $2.61(1.37)$ \\
\hline Ethyne & $2.32(1.41)$ & $-2.46^{* * *}$ & $2.58(1.63)$ & $0.187^{* * *}$ & $0.204(0.112)$ \\
1-Propyne & $0.196(0.108)$ & $-0.129^{* *}$ & $0.244(0.116)$ & $0.105^{* * *}$ & $0.017(0.008)$ \\
1-Butyne & $1.74 \times 10^{-2}$ & $-0.101^{* * *}$ & $0.219(0.007)$ & & \\
\hline
\end{tabular}

${ }^{*}$ denotes $p<0.05$. ${ }^{* *}$ denotes $p<0.01 .{ }^{* * *}$ denotes $p<0.001$. "N/A" indicates that a significant difference was not found.

Higher emissions of alkynes were observed from dung fuels and chulha cookstoves. The latter observation is consistent with the literature showing flaming combustion generates more alkynes (Barrefors and Petersson, 1995; Lee et al., 2005). Chulha cook fires always had higher MCE than angithi cook fires (Table 1), which rely on smoldering combustion. Approximately the same difference in alkyne emissions results from comparing the chulha to the angithi using dung, in relation to using wood versus dung in combination with the chulha. There were two exceptions in stove type for 1-butane $(p=0.055)$ and 2-butane $(p \gg 0.05)$. The former may or may not have a relationship with stove type, while the latter does not. Emissions of some compounds did not show a relationship with either fuel or stove type; they are listed in Table S2.

VOC emissions from Stockwell et al. (2016) are also provided in Table 1 for comparison of VOC EFs. Samples in Stockwell et al. (2016) were collected in April 2015 in and around Kathmandu and the Tarai plains, which border India. While both are EFs from cookstoves using similar fuels, there are differences in the studies that should be noted. Stockwell et al. (2016) collected measurements of simulated cooking in a laboratory and from cooking fires in households; it was not noted in the latter case what meals were cooked. EFs were calculated from similar WAS measurements, but as grab samples in an area of the kitchen away from the fire (as opposed to the time-integrated approach used here). Emissions were assumed to be well mixed in the kitchen prior to sampling. Stockwell et al. (2016) also used a range of stoves, including the traditional single-pot mud stove; open threestone fire; bhuse chulo; and rocket, chimney, and forced draft stoves. "Dung" cook fires sometimes used a combination of fuels, such as wood. Finally, our study also has a larger sample size than Stockwell et al. (2016), with $n=49$ versus $n \approx 10$.

The emission factors for most compounds determined in this study were lower compared to those reported in Table 4 of the Stockwell et al. (2016) paper. Figure S2.1 visually shows that the EFs were generally lower in the present study. In some cases, EFs in this study were an order of magnitude lower, most notably $n$-pentane and $n$-hexane. We also found that our EFs were always higher for dung-chulha compared to brushwood-chulha, which was not always the case in Stockwell et al. (2016). The EFs in Stockwell et al. (2016) could be biased high due to calculations rather than real differences in emissions. For example, ignoring ash and char carbon and using the same carbon content inflates the EFs reported in our paper by $7 \%$ for dung and $24 \%$ for brushwood emissions. However, this is a small percentage compared to the observed differences between EFs between the two measurements. By examining the EFs reported in the supporting information section of Stockwell et al. (2016), we found that the disagreement resulted from the way the final recommended EFs were obtained from the measurements. Because Stockwell et al. (2016) measured both laboratory and field cook fires, they elected to adjust the laboratory EFs to account for the lower MCEs observed in the field. It has been shown by Roden et al. (2009) and Johnson et al. (2008) that cooking activities can strongly influence emissions, for example due to the cook tending to the cook fire differently and thus affecting combustion conditions. However, such adjustments should be done with caution because EF and MCE do not always follow a linear trend, as explained in the next section. Figure S2.2 plots the unadjusted laboratory and field 


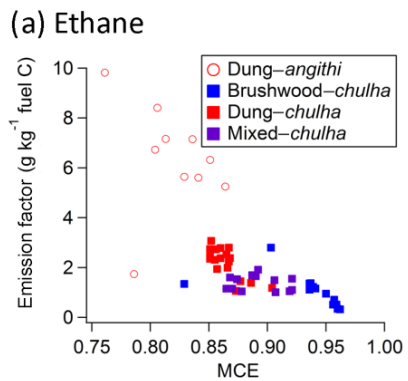

(d) Alkenes (minus ethene)

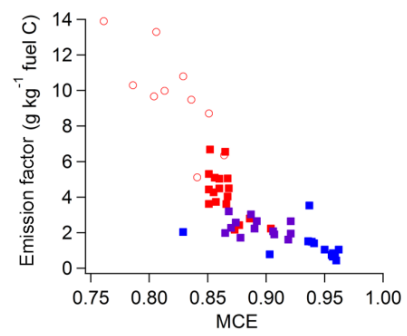

(b) Ethene

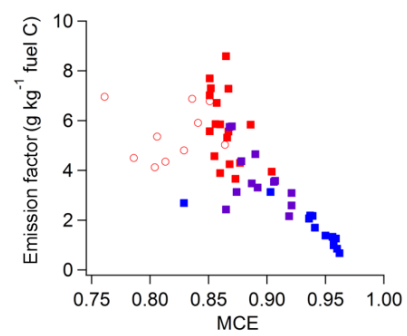

(e) Benzene

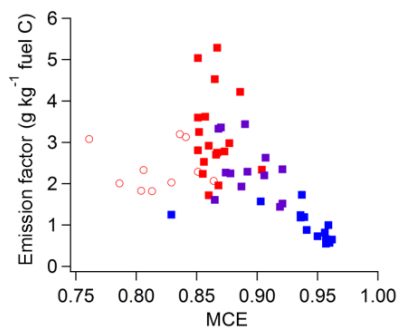

(c) Total alkynes

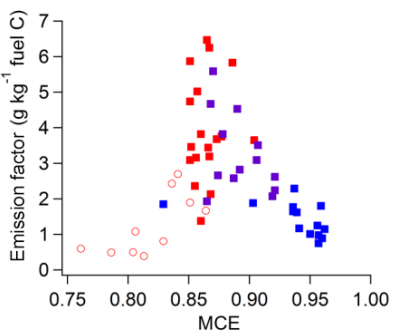

(f) 1,3-Butadiene

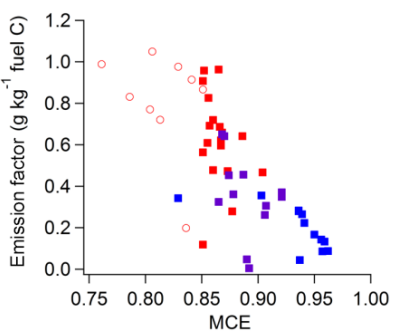

Figure 3. Emission factors as a function of modified combustion efficiency (MCE) for select species. Open circles indicate cooking events conducted with angithi stoves, whereas filled squares indicate chulha stoves. Color indicates fuel: brushwood (blue), dung (red), or mixed (purple). 1-Buten-3-yne is grouped in with alkynes.

emission factors as a function of MCE for all the measurements in Stockwell et al. (2016), as well as this study. Plotting the unadjusted EFs resolves most of the differences in observed EFs between the two studies. The data show an encouraging level agreement despite the differences in the experimental design between the two studies.

\subsection{Modified combustion efficiency}

The use of dung and angithi, rather than brushwood and chulha, respectively, results in lower modified combustion efficiencies as shown in Fig. 3. In general, at lower MCEs we measured higher emissions of gas-phase compounds as discussed in Sect. 3.1. For example, emissions of ethane (Fig. 3a) and other alkanes increase with decreasing MCE. However the dependence of ethane EF on MCE is not linear as observed in previous studies (Liu et al., 2017; Selimovic et al., 2018). For other VOCs, the dependence of the EF on MCE deviates from the linear trend even more, with the maximum EF observed at intermediate MCE values. For example, the ethene EF (Fig. 3b) increases with decreasing MCE at MCEs $>0.85$, but it has the opposite trend at MCEs $<0.85$. Previously, we discussed that there is no relationship between ethene EF and stove type, and we see this more clearly in Fig. 3b. Alkynes have the same relationship to MCE as ethene, but it is even more pronounced (Fig. 3c). Benzene (Fig. 3e) stands apart from other aromatics with a relationship with MCE similar to ethene, while other aromatics have an EF-versus-MCE curve similar to alkanes and most other VOCs. In Fig. 3e, we see again that emissions from brushwood-chulha and dung-angithi cook fires result in lower emissions of benzene compared to dungchulha. Alkenes with two double bonds generally have a negative correlation between emissions and MCE, such as 1,3butadiene in Fig. 3f. The 1,3-butadiene EF-versus-MCE plot is not necessarily representative of all analogous plots for alkenes with two double bonds, as they have different shapes. 1,3-Butadiene was chosen as its emission is high compared to other compounds in its subcategory, and it also has health implications. It also happens to have a more linear relationship with MCE, albeit noisy.

It is of interest to compare EFs obtained from different fuel-stove combinations but with the same MCE. In the case of ethane, different cook fire types yield vastly different EFs at the same MCE. For example, at MCE $\approx 0.87$, mixed fuelchulha has an EF of roughly $1.5 \mathrm{~g} \mathrm{~kg}^{-1}$ fuel $\mathrm{C}$, dung-chulha is $2.5 \mathrm{~g} \mathrm{~kg}^{-1}$ fuel $\mathrm{C}$, and dung-angithi is $5.5 \mathrm{~g} \mathrm{~kg}^{-1}$ fuel $\mathrm{C}$. Knowledge of the cook fire MCE alone is not sufficient to determine the $\mathrm{EF}$ of ethane. Combustion conditions specific to the fuel-stove combination are a significant factor in determining cook fire emissions. A similar conclusion can be reached for most of the measured gases, including nonethene alkenes in Fig. 3d.

\subsection{Secondary pollutant formation and reactivity}

\subsubsection{OH reactivity and ozone-forming potential}

The total $\mathrm{OH}$ reactivity based on the measured VOCs in Fig. $2 b$ is given in units of $\mathrm{s}^{-1} \mathrm{ppbv}^{-1} \mathrm{CO}$. Predicted $\mathrm{OH}$ reactivities in the village due to a single cooking event are 
$10.2,6.73,4.93$, and $4.83 \mathrm{~s}^{-1}$ for emissions from dungangithi, dung-chulha, mixed fuel-chulha, and brushwoodchulha cook fires, respectively. This assumes a CO mixing ratio of $338 \mathrm{ppbv}$, which we measured as the average background mixing ratio over the whole campaign. The relative total $\mathrm{OH}$ reactivity is over twice as high for dung-angithi cook fires as it is for brushwood-chulha cook fires.

The classes of compounds that act as the most important $\mathrm{OH}$ radical sinks in descending order are alkenes, oxygenates, furans, terpenes, and aromatics. Alkenes make up more than $50 \%$ of $\mathrm{OH}$ reactivity for all cook fire types. Ethene (by fuel type) and propene (by fuel and stove combination) are mostly responsible for the differences in fuel-stove combination results for alkenes. For oxygenates, methanol $(p<0.01)$ and acrolein $(p<0.05)$ have significantly higher $\mathrm{OH}$ reactivity with wood fuel, while acetaldehyde has significantly higher $\mathrm{OH}$ reactivity with the angithi stove $(p<0.001)$. Differences in $\mathrm{OH}$ reactivity due to furans were observed for stove type but not fuel type. All three of the measured furans significantly contribute $(p<0.001)$ to a $6 \%$ increase in the fraction of $\mathrm{OH}$ reactivity due to furans for dung-angithi $(12 \%)$ as opposed to dung-chulha (6\%). The percentage of $\mathrm{OH}$ reactivity due to aromatics is constant at $\sim 4 \%$ for the fuel-stove combinations. However, different aromatic compounds are responsible for this $\sim 4 \%$ contribution depending on the cook fire type. Benzene dominates $\mathrm{OH}$ reactivity due to aromatics for chulha cook fires. For angithi cook fires aromatics other than benzene, in particular toluene, dictate the $\mathrm{OH}$ reactivity for aromatics. Isoprene is solely responsible for the differences in $\mathrm{OH}$ reactivity due to terpenes.

Figure $2 \mathrm{~d}$ shows the total ozone-forming potential ( $\mathrm{g} \mathrm{O}_{3} \mathrm{~kg}^{-1}$ fuel) in the MIR scenario, as well as contributions to OFP by compound class. A critical step in photochemical ozone production is VOC reacting with $\mathrm{OH}$. Therefore, the ozone-forming potential contributions by compound class are similar to those for $\mathrm{OH}$ reactivity (Fig. 2c). Total OFP is nearly a factor of 3 higher for dung-chulha compared to brushwood-chulha, while it is twice as high for dungangithi as compared to dung-chulha.

\subsubsection{SOA formation potential}

SOAP-weighted emissions relate SOA production from the different cook fire types in a qualitative manner. The contribution of each compound class to the total SOA formation potential is shown in Fig. 2c. We emphasize that the SOAPweighted emissions are reflective of only the measured VOCs, and there are likely semi-volatile and intermediatevolatility compounds that are not measured but also contribute to SOA formation. The sum of the contributions by each compound class, or the total SOA-forming potential, is also shown below the pie charts. Dung fuels and angithi stoves yield larger amounts of SOA. However, fuel type is more important than stove type in terms of SOA forma- tion. SOAP-weighted emissions are a factor of 3 higher for dung-chulha compared to brushwood-chulha. We discussed previously that benzene emissions are significantly higher from chulha cook fires compared to angithi cook fires. These higher benzene emissions directly impact public health and also dictate SOA formation for chulha emissions. Benzene emissions are responsible for at least half of the SOA formation from the chulha cook fire VOCs we measured. Beyond benzene, aromatics make up on average roughly $95 \%$ of SOA precursors measured in this study for all cook fires. While benzene is prominent for chulha cook fires, $\mathrm{C}_{8}-\mathrm{C}_{9}$ aromatics, toluene, and benzene contribute in approximately equal proportions to SOA formation in dung-angithi smoke plumes.

\section{Atmospheric implications and conclusions}

The extent of ozone formation hinges on the villages' overall $\mathrm{NO}_{x}$ levels as well as VOC emissions. However, in a VOClimited regime, with each household in this village cooking three meals a day using the chulha and mixed fuels (brushwood + dung), $3.3 \times 10^{5} \mathrm{~g}$ ozone per day is expected to be produced due solely to cookstove use. This was estimated based off the Census of India (2011) data for the village of Khatela and assuming the same fuel consumption as that used in this study. Over the lunch hour, when solar radiation is most intense, $30 \mathrm{ppbv}$ of excess ozone is predicted. For this calculation we assumed a calm wind speed of $0.5 \mathrm{~m} \mathrm{~s}^{-1}$ and confined our analysis to the village of Khatela, with a boundary layer height of $1 \mathrm{~km}$ and village length of $1 \mathrm{~km}$. In a similar way we calculated the amount of ozone that could be generated from cooking animal fodder. We assumed that every household prepares animal fodder every 3 days in addition to the assumptions already discussed, resulting in an additional $7.9 \times 10^{4} \mathrm{~g}$ of $\mathrm{O}_{3}$ produced per day from cooking. If we assume every household in the village prepares animal fodder in the same hour, excess ozone levels of $7 \mathrm{ppbv}$ are predicted, using the same assumptions described earlier for the lunch hour. We should note that these estimations are approximate and a regional air quality model with detailed household level inputs should be used to more precisely predict the impact of cook fire emissions on ozone levels.

Using dung patties as opposed to brushwood has a large impact on local $\mathrm{PM}_{2.5}$ and ozone levels. Measured $\mathrm{PM}_{2.5}$ concentrations were more than a factor of 2 higher for dungchulha compared to brushwood-chulha in grams emitted per kilogram of fuel carbon burned. In addition to this, the total SOA-forming potential is 3 times higher for dungchulha than that of brushwood-chulha. We also estimated that dung-chulha cook fires produce roughly 3 times more ozone in the MIR regime than brushwood-chulha cook fires ( $163 \mathrm{~g} \mathrm{O}_{3} \mathrm{~kg}^{-1}$ fuel $\mathrm{C}$ versus $56.9 \mathrm{~g} \mathrm{O}_{3} \mathrm{~kg}^{-1}$ fuel $\mathrm{C}$ ). However, compounds such as benzene are emitted in higher quantities from the chulha $\left(1.03 \mathrm{~g} \mathrm{~kg}^{-1}\right.$ dry fuel) versus angithi 
( $0.373 \mathrm{~g} \mathrm{~kg}^{-1}$ dry fuel), and this public health concern should be investigated in more detail.

Data availability. All relevant data have been included in this publication in the form of tables. The raw data are available in the form of an Excel file in the Supplement.

Supplement. The supplement related to this article is available online at: https://doi.org/10.5194/acp-18-15169-2018-supplement.

Author contributions. RDE, SAN, KRS, and DRB designed the experiments. AP and NKA facilitated the field measurements in India. LTF, RW, and AY collected the samples. LTF, RDE, RW, SM, and SAN contributed to the data analysis. LTF wrote the paper with contribution from all the co-authors.

Competing interests. The authors declare that they have no conflict of interest.

Disclaimer. The contents are solely the responsibility of the authors and do not necessarily represent the official views of the US EPA. The US EPA does not endorse the purchase of any commercial products or services mentioned in the publication.

Acknowledgements. We thank the village of Khatela and our cook for welcoming us and for participating in the study. We also want to acknowledge Sneha Gautam's role in supporting the fieldwork. This research was supported by EPA STAR grant R835425, "Impacts of household sources on outdoor pollution at village and regional scales in India".

Edited by: Eleanor Browne

Reviewed by: two anonymous referees

\section{References}

Balakrishnan, K., Ghosh, S., Ganguli, B., Sambandam, S., Bruce, N., Barnes, D. F., and Smith, K. R.: State and national household concentrations of $\mathrm{PM}_{2.5}$ from solid cookfuel use: Results from measurements and modeling in India for estimation of the global burden of disease, Environ. Health-Glob., 12, 77, https://doi.org/10.1186/1476-069X-12-77, 2013.

Balakrishnan, K., Sambandam, S., Ghosh, S., Mukhopadhyay, K., Vaswani, M., Arora, N. K., Jack, D., Pillariseti, A., Bates, M. N., and Smith, K. R.: Household Air Pollution Exposures of Pregnant Women Receiving Advanced Combustion Cookstoves in India: Implications for Intervention, Ann. Glob. Health, 81, 375385, https://doi.org/10.1016/j.aogh.2015.08.009, 2015.

Barrefors, G. and Petersson, G.: Volatile hydrocarbons from domestic wood burning, Chemosphere, 30, 1551-1556, https://doi.org/10.1016/0045-6535(95)00048-D, 1995.
Bisht, D. S., Srivastava, A. K., Pipal, A. S., Srivastava, M. K., Pandey, A. K., Tiwari, S., and Pandithurai, G.: Aerosol characteristics at a rural station in southern peninsular India during CAIPEEX-IGOC: physical and chemical properties, Environ. Sci. Pollut. R., 22, 5293-5304, https://doi.org/10.1007/s11356014-3836-1, 2015.

Butt, E. W., Rap, A., Schmidt, A., Scott, C. E., Pringle, K. J., Reddington, C. L., Richards, N. A. D., Woodhouse, M. T., RamirezVillegas, J., Yang, H., Vakkari, V., Stone, E. A., Rupakheti, M., S. Praveen, P., G. van Zyl, P., P. Beukes, J., Josipovic, M., Mitchell, E. J. S., Sallu, S. M., Forster, P. M., and Spracklen, D. V.: The impact of residential combustion emissions on atmospheric aerosol, human health, and climate, Atmos. Chem. Phys., 16, 873-905, https://doi.org/10.5194/acp-16-873-2016, 2016.

Carter, W. P. L.: Development of Ozone Reactivity Scales for Volatile Organic Compounds, Air Waste, 44, 881-899, https://doi.org/10.1080/1073161X.1994.10467290, 1994.

Carter, W. P. L.: Development of the SAPRC-07 chemical mechanism, Atmos. Environ., 44, 5324-5335, https://doi.org/10.1016/J.ATMOSENV.2010.01.026, 2010.

Census of India: Households by Availability of Separate Kitchen and Type of Fuel Used for Cooking, available at: http://www. censusindia.gov.in/2011census/Hlo-series/HH10.html (last access: 8 August 2017), 2011.

Chafe, Z. A., Brauer, M., Klimont, Z., Van Dingenen, R., Mehta, S., Rao, S., Riahi, K., Dentener, F. and Smith, K. R.: Household Cooking with Solid Fuels Contributes to Ambient $\mathrm{PM}_{2.5}$ Air Pollution and the Burden of Disease, Environ. Health Persp., 122, 1314-1320, https://doi.org/10.1289/ehp.1206340, 2014.

Chen, Y. and Bond, T. C.: Light absorption by organic carbon from wood combustion, Atmos. Chem. Phys., 10, 1773-1787, https://doi.org/10.5194/acp-10-1773-2010, 2010.

Colman, J. J., Swanson, A. L., Meinardi, S., Sive, B. C., Blake, D. R., and Rowland, F. S.: Description of the analysis of a wide range of volatile organic compounds in whole air samples collected during PEM-Tropics A and B, Anal. Chem., 73, 37233731, https://doi.org/10.1021/ac010027g, 2001.

Conibear, L., Butt, E. W., Knote, C., Arnold, S. R., and Spracklen, D. V.: Residential energy use emissions dominate health impacts from exposure to ambient particulate matter in India, Nat. Commun., 9, 617, https://doi.org/10.1038/s41467-018-02986-7, 2018.

Crutzen, P. J., Heidt, L. E., Krasnec, J. P., Pollock, W. H., and Seiler, W.: Biomass burning as a source of atmospheric gases $\mathrm{CO}, \mathrm{H}_{2}, \mathrm{~N}_{2} \mathrm{O}, \mathrm{NO}, \mathrm{CH}_{3} \mathrm{Cl}$ and $\mathrm{COS}$, Nature, 282, 253-256, https://doi.org/10.1038/282253a0, 1979.

Derwent, R. G., Jenkin, M. E., Saunders, S. M., and Pilling, M. J.: Photochemical ozone creation potentials for organic compounds in northwest Europe calculated with a master chemical mechanism, Atmos. Environ., 32, 2429-2441, https://doi.org/10.1016/S1352-2310(98)00053-3, 1998.

Derwent, R. G., Jenkin, M. E., Utembe, S. R., Shallcross, D. E., Murrells, T. P., and Passant, N. R.: Secondary organic aerosol formation from a large number of reactive manmade organic compounds, Sci. Total Environ., 408, 3374-3381, https://doi.org/10.1016/j.scitotenv.2010.04.013, 2010.

Finlayson-Pitts, B. J. and Pitts, J. N.: Chemistry of the Upper and Lower Atmosphere: Theory, Experiments, and Applications, Academic Press, San Diego, CA, USA, 2000. 
Fleming, L. T., Lin, P., Laskin, A., Laskin, J., Weltman, R., Edwards, R. D., Arora, N. K., Yadav, A., Meinardi, S., Blake, D. R., Pillarisetti, A., Smith, K. R., and Nizkorodov, S. A.: Molecular composition of particulate matter emissions from dung and brushwood burning household cookstoves in Haryana, India, Atmos. Chem. Phys., 18, 2461-2480, https://doi.org/10.5194/acp18-2461-2018, 2018.

GBD MAPS Working Group: Summary for Policy Makers, in: Burden of Disease Attributable to Major Air Pollution Sources in India, Health Effects Institute, Boston, MA, USA, 2018.

Guttikunda, S., Jawahar, P., Gota, S., and KA, N.: UrbanEmissions.info, available at: http://www.urbanemissions.info/ (last access: 10 August 2017), 2016.

Jayarathne, T., Stockwell, C. E., Bhave, P. V., Praveen, P. S., Rathnayake, C. M., Islam, Md. R., Panday, A. K., Adhikari, S., Maharjan, R., Goetz, J. D., DeCarlo, P. F., Saikawa, E., Yokelson, R. J., and Stone, E. A.: Nepal Ambient Monitoring and Source Testing Experiment (NAMaSTE): emissions of particulate matter from wood- and dung-fueled cooking fires, garbage and crop residue burning, brick kilns, and other sources, Atmos. Chem. Phys., 18, 2259-2286, https://doi.org/10.5194/acp18-2259-2018, 2018.

Johnson, M., Edwards, R., Alatorre Frenk, C., and Masera, O.: In-field greenhouse gas emissions from cookstoves in rural Mexican households, Atmos. Environ., 42, 1206-1222, https://doi.org/10.1016/j.atmosenv.2007.10.034, 2008.

Klimont, Z., Kupiainen, K., Heyes, C., Purohit, P., Cofala, J., Rafaj, P., Borken-Kleefeld, J., and Schöpp, W.: Global anthropogenic emissions of particulate matter including black carbon, Atmos. Chem. Phys., 17, 8681-8723, https://doi.org/10.5194/acp-178681-2017, 2017.

Lee, S., Baumann, K., Schauer, J. J., Sheesley, R. J., Naeher, L. P., Meinardi, S., Blake, D. R., Edgerton, E. S., Russell, A. G., and Clements, M.: Gaseous and particulate emissions from prescribed burning in Georgia, Environ. Sci. Technol., 39, 90499056, https://doi.org/10.1021/es0515831, 2005.

Lelieveld, J., Evans, J. S., Fnais, M., Giannadaki, D., and Pozzer, A.: The contribution of outdoor air pollution sources to premature mortality on a global scale, Nature, 525, 367-371, https://doi.org/10.1038/nature15371, 2015.

Liu, X., Huey, L. G., Yokelson, R. J., Selimovic, V., Simpson, I. J., Müller, M., Jimenez, J. L., Campuzano-Jost, P., Beyersdorf, A. J., Blake, D. R., Butterfield, Z., Choi, Y., Crounse, J. D., Day, D. A., Diskin, G. S., Dubey, M. K., Fortner, E., Hanisco, T. F., Hu, W., King, L. E., Kleinman, L., Meinardi, S., Mikoviny, T., Onasch, T. B., Palm, B. B., Peischl, J., Pollack, I. B., Ryerson, T. B., Sachse, G. W., Sedlacek, A. J., Shilling, J. E., Springston, S., St. Clair, J. M., Tanner, D. J., Teng, A. P., Wennberg, P. O., Wisthaler, A., and Wolfe, G. M.: Airborne measurements of western U.S. wildfire emissions: Comparison with prescribed burning and air quality implications, J. Geophys. Res.-Atmos., 122, 6108-6129, https://doi.org/10.1002/2016JD026315, 2017.

Manion, J. A., Huie, R. E., Levin, R. D., Burgess Jr., D. R., Orkin, V. L., Tsang, W., McGivern, W. S., Hudgens, J. W., Knyazev, V. D., Atkinson, D. B., Chai, E., Tereza, A. M., Lin, C. Y., Allison, T. C., Mallard, W. G., Westley, F., Herron, J. T., Hampson, R. F., and Frizzell, D. H.: NIST Chemical Kinetics Database, in: NIST Standard Reference Database 17, National Institute of Standards and Technology, Gaithersburg, Maryland, USA, 20899-8320, 2015.

Mukhopadhyay, R., Sambandam, S., Pillarisetti, A., Jack, D., Mukhopadhyay, K., Balakrishnan, K., Vaswani, M., Bates, M. N., Kinney, P. L., Arora, N., and Smith, K. R.: Cooking practices, air quality, and the acceptability of advanced cookstoves in Haryana, India: an exploratory study to inform large-scale interventions, Global Health Action, 5, 1-13, https://doi.org/10.3402/gha.v5i0.19016, 2012.

Ojha, N., Naja, M., Singh, K. P., Sarangi, T., Kumar, R., Lal, S., Lawrence, M. G., Butler, T. M., and Chandola, H. C.: Variabilities in ozone at a semi-urban site in the IndoGangetic Plain region: Association with the meteorology and regional processes, J. Geophys. Res.-Atmos., 117, D20301, https://doi.org/10.1029/2012JD017716, 2012.

Pandey, A., Sadavarte, P., Rao, A., and Venkataraman, C.: Trends in multi-pollutant emissions from a technologylinked inventory for India: II. Residential, agricultural and informal industry sectors, Atmos. Environ., 99, 341-352, https://doi.org/10.1016/J.ATMOSENV.2014.09.080, 2014.

Pankow, J. F.: An absorption model of the gas/aerosol partitioning involved in the formation of secondary organic aerosol, Atmos. Environ., 28, 189-193, https://doi.org/10.1016/13522310(94)90094-9, 1994.

Pillarisetti, A., Vaswani, M., Jack, D., Balakrishnan, K., Bates, M. N., Arora, N. K., and Smith, K. R.: Patterns of Stove Usage after Introduction of an Advanced Cookstove: The Long-Term Application of Household Sensors, Environ. Sci. Technol., 48, 1452514533, https://doi.org/10.1021/es504624c, 2014.

Reddy, B. S. K.: Analysis of Diurnal and Seasonal Behavior of Surface Ozone and Its Precursors $\left(\mathrm{NO}_{x}\right)$ at a Semi-Arid Rural Site in Southern India, Aerosol Air Qual. Res., 12, 1081-1094, https://doi.org/10.4209/aaqr.2012.03.0055, 2012.

Roden, C. A. and Bond, T. C.: Emission Factors and Real-Time Optical Properties of Particles Emitted from Traditional Wood Burning Cookstoves, Environ. Sci. Technol., 40, 6750-6757, https://doi.org/10.1021/ES052080I, 2006.

Roden, C. A., Bond, T. C., Conway, S., Osorto Pinel, A. B., MacCarty, N., and Still, D.: Laboratory and field investigations of particulate and carbon monoxide emissions from traditional and improved cookstoves, Atmos. Environ., 43, 11701181, https://doi.org/10.1016/j.atmosenv.2008.05.041, 2009.

RStudio Team: RStudio: Integrated Development Environment for R, RStudio, Inc, Boston, MA, USA, available at: http://www. rstudio.com/ (last access: 18 October 2018), 2016.

Selimovic, V., Yokelson, R. J., Warneke, C., Roberts, J. M., de Gouw, J., Reardon, J., and Griffith, D. W. T.: Aerosol optical properties and trace gas emissions by PAX and OP-FTIR for laboratory-simulated western US wildfires during FIREX, Atmos. Chem. Phys., 18, 2929-2948, https://doi.org/10.5194/acp18-2929-2018, 2018.

Silva, R. A., Adelman, Z., Fry, M. M., and West, J. J.: The Impact of Individual Anthropogenic Emissions Sectors on the Global Burden of Human Mortality due to Ambient Air Pollution, Environ. Health Persp., 124, 1776-1784, https://doi.org/10.1289/EHP177, 2016.

Simpson, I. J., Aburizaiza, O. S., Siddique, A., Barletta, B., Blake, N. J., Gartner, A., Khwaja, H., Meinardi, S., Zeb, J., and Blake, D. R.: Air Quality in Mecca and Surrounding Holy Places in 
Saudi Arabia During Hajj: Initial Survey, Environ. Sci. Technol., 48, 8529-8537, https://doi.org/10.1021/es5017476, 2014.

Smith, K. R., Khalil, M. A. K., Rasmussen, R. A., Thorneloe, S. A., Manegdeg, F., and Apte, M.: Greenhouse gases from biomass and fossil fuel stoves in developing countries: A Manila pilot study, Chemosphere, 26, 479-505, https://doi.org/10.1016/00456535(93)90440-G, 1993.

Smith, K. R., Uma, R., Kishore, V., Lata, K., Joshi, V., Zhang, J., Rasmussen, R., and Khalil, M.: Greenhouse gases from smallscale combustion devices in developing countries, phase IIA. Household stoves in India (EPA/600/R-00/052), U.S. Environmental Protection Agency, Washington, D.C., USA, 2000a.

Smith, K. R., Uma, R., Kishore, V. V. N., Zhang, J., Joshi, V., and Khalil, M. A. K.: Greenhouse Implications of Household Stoves: An Analysis for India, Annu. Rev. Energ. Env., 25, 741-763, 2000b.

Stockwell, C. E., Christian, T. J., Goetz, J. D., Jayarathne, T., Bhave, P. V., Praveen, P. S., Adhikari, S., Maharjan, R., DeCarlo, P. F., Stone, E. A., Saikawa, E., Blake, D. R., Simpson, I. J., Yokelson, R. J., and Panday, A. K.: Nepal Ambient Monitoring and Source Testing Experiment (NAMaSTE): emissions of trace gases and light-absorbing carbon from wood and dung cooking fires, garbage and crop residue burning, brick kilns, and other sources, Atmos. Chem. Phys., 16, 11043-11081, https://doi.org/10.5194/acp-16-11043-2016, 2016.
Surawski, N. C., Sullivan, A. L., Meyer, C. P., Roxburgh, S. H., and Polglase, P. J.: Greenhouse gas emissions from laboratoryscale fires in wildland fuels depend on fire spread mode and phase of combustion, Atmos. Chem. Phys., 15, 5259-5273, https://doi.org/10.5194/acp-15-5259-2015, 2015.

Tihay-Felicelli, V., Santoni, P. A., Gerandi, G., and Barboni, T.: Smoke emissions due to burning of green waste in the Mediterranean area: Influence of fuel moisture content and fuel mass, Atmos. Environ., 159, 92-106, https://doi.org/10.1016/J.ATMOSENV.2017.04.002, 2017.

Tsai, S. M., Zhang, J., Smith, K. R., Ma, Y., Rasmussen, R. A., and Khalil, M. A. K.: Characterization of Nonmethane Hydrocarbons Emitted from Various Cookstoves Used in China, Environ. Sci. Technol., 37, 2869-2877, https://doi.org/10.1021/es026232a, 2003.

Wathore, R., Mortimer, K., and Grieshop, A. P.: In-Use Emissions and Estimated Impacts of Traditional, Natural- and Forced-Draft Cookstoves in Rural Malawi, Environ. Sci. Technol., 51, 19291938, https://doi.org/10.1021/acs.est.6b05557, 2017.

Yevich, R. and Logan, J. A.: An assessment of biofuel use and burning of agricultural waste in the developing world, Global Biogeochem. Cy., 17, 1095, https://doi.org/10.1029/2002GB001952, 2003.

Zhang, J., Smith, K., Ma, Y., Ye, S., Jiang, F., Qi, W., Liu, P., Khalil, M. A., Rasmussen, R., and Thorneloe, S.: Greenhouse gases and other airborne pollutants from household stoves in China: a database for emission factors, Atmos. Environ., 34, 4537-4549, https://doi.org/10.1016/S1352-2310(99)00450-1, 2000. 